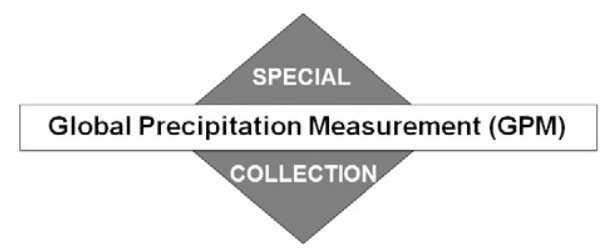

\title{
Sensitivity of Simulated GMI Brightness Temperatures to Variations in Particle Size Distributions in a Severe Hailstorm
}

\author{
KENNETH D. LEPPERT II \\ University of Louisiana at Monroe, Monroe, Louisiana \\ DANIEL J. CECIL \\ NASA Marshall Space Flight Center, Huntsville, Alabama
}

(Manuscript received 4 February 2019, in final form 15 June 2019)

\begin{abstract}
Global Precipitation Measurement (GPM) Microwave Imager (GMI) brightness temperatures (BTs) were simulated over a case of severe convection in Texas using ground-based S-band radar and the Atmospheric Radiative Transfer Simulator. The median particle diameter $D_{o}$ of a normalized gamma distribution was varied for different hydrometeor types under the constraint of fixed radar reflectivity to better understand how simulated GMI BTs respond to changing particle size distribution parameters. In addition, simulations were conducted to assess how low BTs may be expected to reach from realistic (although extreme) particle sizes or concentrations. Results indicate that increasing $D_{o}$ for cloud ice, graupel, and/or hail leads to warmer BTs (i.e., weaker scattering signature) at various frequencies. Channels at 166.0 and $183.31 \pm 7 \mathrm{GHz}$ are most sensitive to changing $D_{o}$ of cloud ice, channels at $\geq 89.0 \mathrm{GHz}$ are most sensitive to changing $D_{o}$ of graupel, and at 18.7 and $36.5 \mathrm{GHz}$ they show the greatest sensitivity to hail $D_{o}$. Simulations contrasting BTs above high concentrations of small (0.5-cm diameter) and low concentrations of large (20-cm diameter) hailstones distributed evenly across a satellite pixel showed much greater scattering using the higher concentration of smaller hailstones with BTs as low as $\sim 110, \sim 33, \sim 22, \sim 46, \sim 100$, and $\sim 106 \mathrm{~K}$ at 10.65, 18.7, 36.5, 89.0, 166.0, and $183.31 \pm 7 \mathrm{GHz}$, respectively. These results suggest that number concentration is more important for scattering than particle size given a constant S-band radar reflectivity.
\end{abstract}

\section{Introduction}

Numerous spaceborne passive microwave instruments have been used for precipitation estimates including the Electrically Scanning Microwave Radiometer (e.g., Wilheit et al. 1977; one of the earliest instruments) up to the current suite of instruments involved with the Global Precipitation Measurement (GPM) mission (Hou et al. 2014; Skofronick-Jackson et al. 2017). The Core Observatory along with several additional constellation satellites make up the GPM mission. The radiometer on board the Core Observatory [i.e., GPM Microwave Imager (GMI)] senses radiation at various frequencies from 10.65 to $183.31 \pm 7 \mathrm{GHz}$ (Hou et al. 2014). Note that for succinctness hereafter, all frequencies will be referred to by their integer value (e.g., $10.65 \mathrm{GHz}$ will be referred to as $10 \mathrm{GHz}$ ).

Corresponding author: Kenneth Leppert II, leppert@ulm.edu
There are two basic physical processes involved in passive microwave precipitation retrieval. The first process is based on the emission of radiation by liquid hydrometeors (e.g., Wilheit et al. 1991). Specifically, the emissivity of liquid hydrometeors is greater than that of the ocean surface, so warmer brightness temperatures (BTs) are measured above liquid precipitation than above a precipitation-free ocean surface (e.g., Spencer et al. 1989; Adler et al. 1991; Wilheit et al. 1991; McGaughey et al. 1996). The emission signal from liquid precipitation depends on several factors including characteristics of the background, frequency, liquid mass, rain rate, and thickness of the liquid layer (e.g., Wilheit et al. 1991; Ferraro and Marks 1995; McGaughey et al. 1996). A lower surface emissivity allows for a stronger contrast between the background surface and the emission from the liquid precipitation. An increase in liquid mass, rain rate, and/or layer thickness results in greater emission. 
The second basic process involved in passive microwave precipitation retrieval is scattering by hydrometeors (e.g., Ferraro and Marks 1995). In particular, precipitation-sized ice hydrometeors scatter some of the upwelling radiation, reducing the BTs (e.g., Spencer et al. 1983; Spencer and Santek 1985; Spencer et al. 1989; Smith et al. 1992; Ferraro and Marks 1995). Scattering depends on several factors including microwave frequency, particle phase, density, size, shape, concentration, and vertical distribution (e.g., Vivekanandan et al. 1991; Bennartz and Petty 2001; Kim et al. 2007; Meirold-Mautner et al. 2007; Galligani et al. 2013; Tang et al. 2017). Absorption/emission by ice is negligibly small (Casella et al. 2008), thus scattering becomes the main effect from ice hydrometeors (e.g., Spencer et al. 1989; Bennartz and Bauer 2003; Galligani et al. 2013). A greater optical depth of ice results in greater scattering (lower BTs; e.g., Meirold-Mautner et al. 2007; Galligani et al. 2013; Olson et al. 2016). In general, scattering increases with increasing particle size without assuming a fixed mass (Galligani et al. 2013). Bennartz and Petty (2001) found that particle size is more important than density in terms of its impact on the resulting BTs.

Bennartz and Bauer (2003) and Hong et al. (2005) examined the sensitivity of high-frequency $(85-183 \mathrm{GHz})$ channels to ice scattering and various environmental parameters (e.g., surface emissivity). Bennartz and Bauer (2003) simulated BTs over several cases of highlatitude weather, whereas Hong et al. (2005) simulated BTs over a case of deep convection in the tropics. Both studies found that channels near 85 and $150 \mathrm{GHz}$ appear useful for the detection of snow, and the response to snow and/or graupel increases with increasing frequency. Hong et al. (2005) found that these highfrequency channels are most sensitive to the presence of graupel, followed by cloud ice and snow.

The assumption of spherical particles greatly simplifies radiative transfer simulations but is not realistic for many frozen particles. Olson et al. (2016) were able to match $165-\mathrm{GHz}$ BT measurements better when using aggregates rather than spherical ice particles. Kulie et al. (2010) and Hogan et al. (2017) similarly found that simulations using spherical particles were unable to match observations at $165 \mathrm{GHz}$. However, Hogan et al. (2017) found that for particles smaller than the wavelength of the radiation, oblate spheroid mixtures of ice and air did produce reasonably realistic results.

This study seeks to better understand the relative importance of hydrometeor type, size, and concentration for passive microwave satellite measurements by using the Atmospheric Radiative Transfer Simulator (ARTS) to simulate GMI BTs (from 10 through $183 \mathrm{GHz}$ ) over a hailstorm. Ground-based, polarimetric S-band radar data are used to define the three-dimensional structure of radar reflectivity $Z_{h}$ and to guide the assumptions of particle type. Complex particle shapes are not considered; only spheroids are modeled. The study seeks to answer three main questions. First, assuming fixed S-band $Z_{h}$, how do BTs respond to changing particle size distribution (PSD) parameters? Second, what is physically causing some measured BTs to be so low, such as those observed in Marra et al. (2017) and Cecil and Chronis (2018)? Finally, in this simplified modeling framework, how low would BTs be expected to reach from extremely large particle sizes (e.g., a few 20-cm hailstones) compared to higher concentrations of smaller particles? A $20-\mathrm{cm}$ maximum diameter is used here because that is near the record observed hailstone size (National Weather Association 2010; Allen et al. 2017). The presence of hail/large ice presents a challenging situation in which to retrieve precipitation estimates from spaceborne passive microwave instruments (Grecu et al. 2016). Thus, the ultimate aim of this work is to better understand the signature of hail/large ice in these measurements in order to better account for these hydrometeor species and improve rainfall estimates and hazardous storm identification when they are present.

\section{Methodology}

For this study, GMI BTs were simulated for a case of severe convection (i.e., numerous reports of hail $>2.5-\mathrm{cm}$ diameter; Storm Prediction Center 2015) near the Dallas-Fort Worth, Texas, Weather Surveillance Radar1988 Doppler (KFWS) from 26 May 2015. Level 2 KFWS data (National Centers for Environmental Information 2015) were obtained for the volume scan beginning at 2225 UTC. This scan best matched in time a GMI overpass. Then the Radx software package developed by the National Centers for Atmospheric Research (https:// ral.ucar.edu/projects/titan/docs/radial_formats/radx.html) was used to convert the dual-polarization radar data from its native polar coordinates to a Cartesian coordinate system. The Cartesian grid is centered on active convection stretching across $32.33^{\circ}-34.59^{\circ} \mathrm{N}, 99.76^{\circ}-97.03^{\circ} \mathrm{W}$ $(251 \times 251$ grid points $)$ with a horizontal and vertical resolution (1-18 km above ground level) of $1 \mathrm{~km}$. Figure 1a shows a map of gridded KFWS $Z_{h}$ from a height of $4 \mathrm{~km}$.

Dolan and Rutledge (2009) and Dolan et al. (2013) developed a fuzzy-logic hydrometeor identification (HID) that we applied to the gridded KFWS data. This HID incorporates temperature (derived from the Fort Worth sounding valid 0000 UTC 27 May 2015) and 


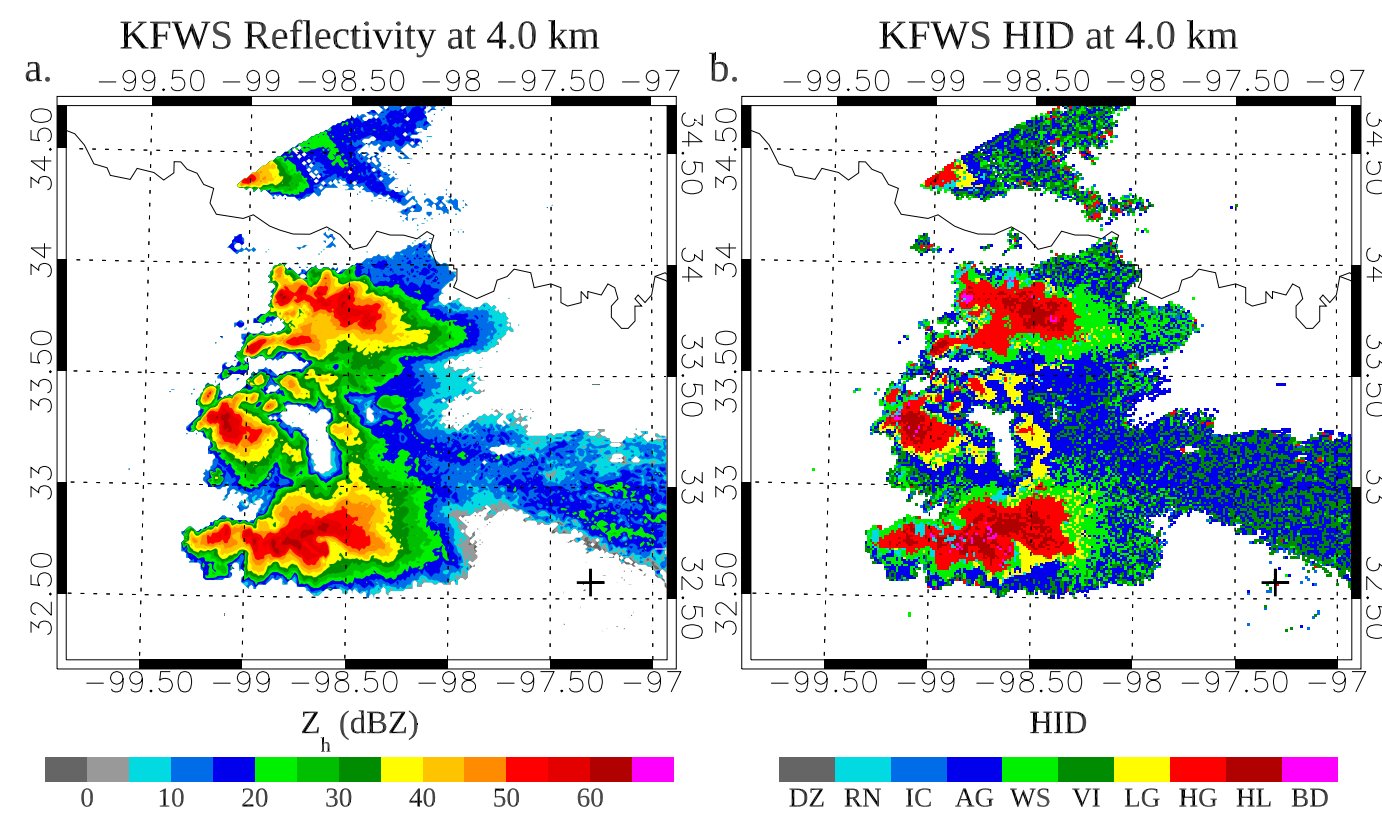

FIG. 1. (a) Gridded reflectivity and (b) the associated HID from KFWS valid 2225 UTC 26 May 2015 at a height of $4 \mathrm{~km}$ (about $-2^{\circ} \mathrm{C}$ temperature). The hydrometeor types are drizzle (DZ), rain (RN), ice crystals (IC), aggregates (AG), wet snow (WS), vertically aligned ice (VI), low-density graupel (LG), high-density graupel (HG), hail (HL), and big drops $(\mathrm{BD})$. The black cross indicates the location of the radar.

four radar variables including $Z_{h}$, differential reflectivity, specific differential phase, and correlation coefficient. Ten possible hydrometeor types are output by the HID including three liquid hydrometeor types (i.e., drizzle, rain, and big drops), four smaller frozen species (i.e., ice crystals, aggregates, wet snow, and vertically oriented ice), and three larger ice species [i.e., low-density graupel (density $\leq 0.55 \mathrm{~g} \mathrm{~cm}^{-3}$ ), high-density graupel (density $>0.55 \mathrm{~g} \mathrm{~cm}^{-3}$ ), and hail]. The HID generates scores (beta function values) at each grid point for each combination of observed variable and hydrometeor type. These scores indicate how well each measurement is consistent with each hydrometeor type. Then the score for each observation is combined into a single value for each hydrometeor species at each grid point. The hydrometeor type with the highest combined score was initially assigned to each radar grid box. For a more detailed description of how this HID was applied, please see Leppert and Cecil (2015). An example of the results from the HID at a height of $4 \mathrm{~km}$ (about $-2^{\circ} \mathrm{C}$ temperature) is shown in Fig. 1b. Hail and high-density graupel are associated with the largest $Z_{h}$ values $(>45 \mathrm{~dB} Z)$ as expected, while lower $Z_{h}$ values correspond with other frozen species.

ARTS is a flexible software package that is capable of simulating three-dimensional radiative transfer primarily in the microwave to infrared parts of the spectrum using a variety of different instrument geometries
(Eriksson et al. 2011; Buehler et al. 2018). ARTS can handle scattering via the discrete ordinate iterative method or Monte Carlo integration. Monte Carlo integration is used here because it is more appropriate for threedimensional calculations. The background atmosphere for our simulations was derived from the Fort Worth sounding valid 0000 UTC 27 May 2015. Specifically, the water vapor mixing ratio was calculated from the sounding measurements and interpolated in the vertical to each radar grid level. Interpolated temperature values from the HID were also used for the ARTS simulations. Thus, the model atmosphere was homogeneous in the horizontal direction. In addition to GMI, the GPM Core Observatory also carries the Dual-Frequency Precipitation Radar (DPR), and surface reflectance information for the ARTS simulations was derived from the surface emissivity values contained in the combined DPR-GMI product (2BCMB; NASA 2016; Olson and Masunaga 2018). The GMI sensor geometry was obtained from the 1B GMI file (orbit number 7052; NASA 2016). Observed BTs used to compare with the simulated values were also extracted from the 1B GMI file. Note that all BTs shown here are horizontally polarized except for $183 \mathrm{GHz}$, which is vertically polarized on GMI (Hou et al. 2014).

Prior to running an ARTS simulation, single-scattering properties have to be calculated separately. The PyARTS software package was used for this purpose. This package calculates the phase matrix, extinction matrix, 
TABLE 1. Fixed parameters used for the single-scattering calculations for different hydrometeor types. The parameters include density $\rho$, orientation, aspect ratio, the minimum and maximum sizes, and the number of size bins between the minimum and maximum sizes. Hydrometeors are assumed to be oriented completely randomly (random) or with the long axis oriented in the horizontal direction (horizontal).

\begin{tabular}{lclccrr}
\hline \hline Hydrometeor type & $\rho\left(\mathrm{g} \mathrm{cm}^{-3}\right)$ & Orientation & Aspect ratio & Min size $(\mathrm{mm})$ & Max size $(\mathrm{mm})$ & No. of bins \\
\hline Rain & 1.000 & Random & 1.0 & 0.10 & 8.00 & 1.60 \\
Cloud ice & 0.900 & Horizontal & 4.8 & 0.05 & 13 & 13 \\
Snow & 0.200 & Horizontal & 2.0 & 0.50 & 1.00 & 12 \\
Low-density graupel & 0.400 & Random & 1.4 & 1.00 & 1000 \\
High-density graupel & 0.725 & Random & 1.4 & 5.00 & 50.00 & 16 \\
Hail & 0.810 & Random & 1.3 & 5.00 & 14.00 \\
Big drops & 1.000 & Random & 1.0 & 9 \\
\hline
\end{tabular}

and absorption vector given fixed properties for each hydrometeor type using T-matrix scattering (Mishchenko 1991, 2000; Mishchenko and Travis 1998). For the simulations, rain and drizzle from the HID were combined into a single rain category, ice crystals and vertically oriented ice were combined into a single cloud ice category, and aggregates and wet snow were combined into a single snow category. The specified parameters for the resulting seven hydrometeor types used for PyARTS following Dolan and Rutledge (2009) and Dolan et al. (2013) are provided in Table 1. The width of each size bin is not constant but gets larger as size is increased, and all hydrometeor species are treated as spheroids. The wavelength of the $183-\mathrm{GHz}$ channel is $1.64 \mathrm{~mm}$ (smallest wavelength), and the maximum size of the cloud ice category is $1.6 \mathrm{~mm}$ (Table 1). Hence, because all cloud ice modeled here are smaller than the wavelength of radiation, the assumption of spheroid ice crystals should provide reasonably realistic results (Hogan et al. 2017). The maximum size of snow particles (12 mm) is larger than the wavelength of several GMI channels, but the simulations used here show relatively little sensitivity to the presence of snow (as shown in section 4).

Prior to calculating PSDs for the various hydrometeor species, snow and cloud ice were removed from radar grid points with a temperature $>280 \mathrm{~K}$. These snow and ice categories at warm temperatures generally resulted from ground clutter. Then the normalized gamma distribution (Olson and Masunaga 2018; Grecu et al. 2016) was used for the PSD of each hydrometeor type. This distribution is given by

$$
n(D)=N_{w} f(\mu)\left(\frac{D}{D_{o}}\right)^{\mu} \exp \left[\frac{-(3.67+\mu)}{D_{o}} D\right],
$$

where

$$
f(\mu)=\frac{6(3.67+\mu)^{\mu+4}}{3.67^{4} \Gamma(\mu+4)},
$$

and $n(D)$ is the spectral number density of particles with diameter $D, N_{w}$ is the intercept of the normalized distribution, $D_{o}$ is the median size diameter, and $\mu$ is the distribution shape factor. For each simulation (listed in Table 2), $D_{o}$ and $\mu$ were specified, and then $N_{w}$ was calculated at each grid point such that the $Z_{h}$ for the resulting PSD matched that observed from KFWS. Specifically, $N_{w}$ was calculated from a lookup table using linear interpolation and the specified $D_{o}, \mu$, and observed $Z_{h}$ values. For the construction of the lookup table, $Z_{h}$ was calculated by integrating across the PSD because ARTS is currently not capable of simulating that parameter (Buehler et al. 2018). The values of $D_{o}$ and $\mu$ as a function of hydrometeor species for the first two simulations (Single_HID and Multi_HID) are given in Table 3. Simulations using different values of $\mu$ suggested little sensitivity to this parameter, so the focus here is on simulations with contrasting $D_{o}$.

Single_HID and Multi_HID are both essentially control simulations. For Single_HID, all the $Z_{h}$ at a given grid point is assumed to result from the single hydrometeor species with the highest combined score from the Dolan and Rutledge (2009) and Dolan et al. (2013) HID algorithm. For Multi_HID, those combined scores are used to weight the contributions from multiple particle types at the same grid point. The Increase_CI_D0, Increase_Snow_D0, Increase_ HG_D0, and Increase_Hail_D0 simulations test the sensitivity to increasing particle size for certain hydrometeor types individually. Based on results from those simulations, Adjust_Ice_D0 increases the size of cloud ice particles and decreases the size of graupel and hail in an attempt to better match the observations. The 0.5 and $20-\mathrm{cm}$ hail simulations test the effects of extremely small and extremely large hail sizes, using monodisperse distributions.

\section{Control simulations}

Maps of the simulated BTs for Single_HID for various frequencies are provided in the top rows of Figs. 2-4. The corresponding observed BTs are given in the bottom 
TABLE 2. List of simulations discussed in the text with their corresponding description.

\begin{tabular}{ll}
\hline \multicolumn{1}{c}{ Simulation } & \multicolumn{1}{c}{ Description } \\
\hline Single_HID & Single hydrometeor type assumed at each grid box (see Table 3 for $\mu$ and $D_{o}$ ) \\
Multi_HID & Multiple hydrometeor types allowed at each grid box (control; see Table 3 for $\mu$ and $D_{o}$ ) \\
Increase_CI_D0 & Increase $D_{o}$ of cloud ice to $0.4 \mathrm{~mm}$ \\
Increase_Snow_D0 & Increase $D_{o}$ of snow to $2.0 \mathrm{~mm}$ \\
Increase_HG_D0 & Increase $D_{o}$ of high-density graupel to $4.5 \mathrm{~mm}$ \\
Increase_Hail_D0 & Increase $D_{o}$ of hail to $18 \mathrm{~mm}$ \\
Adjust_Ice_D0 & Changed $D_{o}$ of cloud ice, high-density graupel, low-density graupel, and hail (see Table 3 for $\mu$ and $D_{o}$ ) \\
0.5-cm hail & All hail is assigned $0.5-\mathrm{cm}$ diameter (monodisperse) \\
20-cm hail & All hail is assigned 20-cm diameter (monodisperse) \\
\hline
\end{tabular}

rows of those same figures. The minimum valid ${ }^{1} \mathrm{BT}$ in each panel of Figs. 2-4 is given at the end of the title of each panel. At 183 and 166 GHz (Fig. 2), the Single_HID simulated BTs in the anvil regions are much lower relative to observed $\mathrm{BTs}$, in some cases by more than $100 \mathrm{~K}$. Minimum simulated BTs at these frequencies are $\sim 50 \mathrm{~K}$ lower than corresponding minimum observed BTs.

In contrast, over the core of the southern convective cell, simulated BTs get warmer than $150 \mathrm{~K}$ at both 183 and $166 \mathrm{GHz}$ where observed BTs are $\sim 90 \mathrm{~K}$. Figure $1 \mathrm{~b}$ indicates that this area contains hail and high-density graupel. Hence, the simulated scattering signature of these large ice species is too weak. We will refer to this area of relatively warm simulated BTs above hail as the "hail hole."

Observed and simulated BTs from Single_HID are shown for 89 and $36 \mathrm{GHz}$ in Fig. 3. Consistent with what is observed over the hail hole at the two highest frequencies, minimum simulated BTs at both 89 and $36 \mathrm{GHz}$ in the convective cores are too warm. In addition, the area of strongest scattering over the three convective cells at $89 \mathrm{GHz}$ appears to be too small relative to that observed from GMI.

A comparison between simulated 18- and 10-GHz BTs and the corresponding observed BTs (Fig. 4) shows that the simulated scattering is generally too strong in the three main convective cells, but the $18-\mathrm{GHz}$ values in the southern cell seem to be closest to observed values. In addition, the simulated values at the two lowest frequencies appear much noisier than the smoothed appearance of the corresponding observed BTs and the simulated BTs at higher frequencies (Figs. 2 and 3). The smoothed appearance of the observed data is due to the lower resolution of these lower-frequency channels (Hou et al. 2014), but the resolution of these channels

\footnotetext{
${ }^{1}$ One simulated pixel had BTs $<5 \mathrm{~K}$ at all frequencies, which were obviously in error and not included in these or subsequent figures.
}

in ARTS was set to be the same as the GMI instrument. Initially, it was thought that the relatively highresolution surface emissivity values (equivalent to the spatial resolution of the DPR of $5 \mathrm{~km}$ ) from the $2 \mathrm{BCMB}$ product may be causing this noisy appearance of the simulated BTs. To test this, the Single HID simulation was repeated except that the surface reflectance was set to the single average value across the entire domain. Results of this simulation (not shown) still resulted in a noisy appearance of simulated BTs at the two lowest frequencies. Thus, it is not clear what is causing this effect.

One possible reason for the discrepancies between simulated and observed BTs is that only a single hydrometeor species is allowed at each model grid point for the Single_HID simulation, while the real atmosphere likely has multiple types at each point. To better account for this, we used the beta function values from the HID to assign portions of the observed $Z_{h}$ to multiple hydrometeor species at each grid volume in the Multi_HID simulation. This procedure can best be illustrated with an example. Assume one particular grid point has a $Z_{h}$ of $50 \mathrm{dBZ}\left(1 \times 10^{5} \mathrm{~mm}^{6} \mathrm{~m}^{-3}\right)$ where hail has a combined beta value of 3 , high-density graupel a beta value of 2 , and all other hydrometeor species have a beta value of 0 . In this case, $3 / 5(60 \%)$

TABLE 3. PSD parameters $\left(\mu\right.$ and $D_{o}$ ) for the Single_HID and Adjust_Ice_D0 simulations as a function of hydrometeor type. The parameters for Multi_HID are identical to Single_HID.

\begin{tabular}{lccccc}
\hline \hline & \multicolumn{2}{c}{$\begin{array}{c}\text { Single_HID/ } \\
\text { Multi_HID }\end{array}$} & & \multicolumn{2}{c}{ Adjust_Ice_D0 } \\
\cline { 2 - 3 } Hydrometeor type & $\mu$ & $D_{o}(\mathrm{~mm})$ & & $\mu$ & $D_{o}(\mathrm{~mm})$ \\
\hline Rain & 0 & 0.75 & & 0 & 0.75 \\
Cloud ice & 0 & 0.20 & & 0 & 0.40 \\
Snow & 0 & 0.50 & & 0 & 0.50 \\
Low-density graupel & 2 & 2.50 & & 2 & 1.50 \\
High-density graupel & 2 & 2.50 & & 2 & 2.00 \\
Hail & 2 & 6.00 & & 2 & 5.50 \\
Big drops & 0 & 6.00 & & 0 & 6.00 \\
\hline
\end{tabular}


a. $\quad$ Single_HID $183.3 \pm 7-\mathrm{GHz}(31.7 \mathrm{~K})$

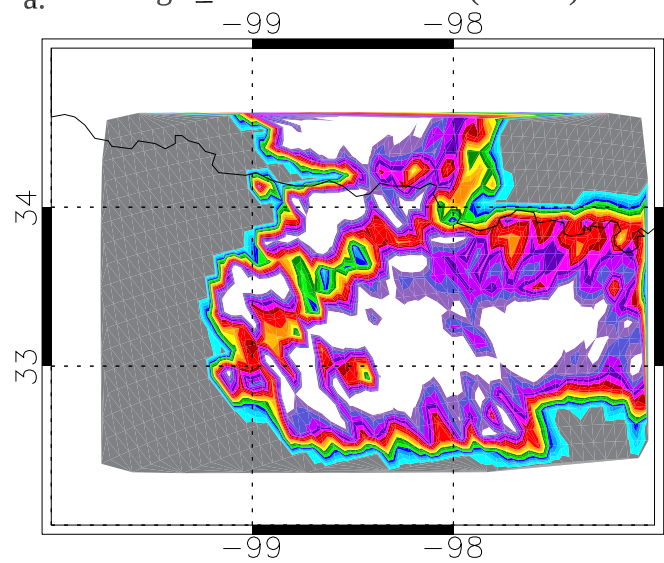

b. Multi_HID $183.3 \pm 7-\mathrm{GHz}(50.4 \mathrm{~K})$

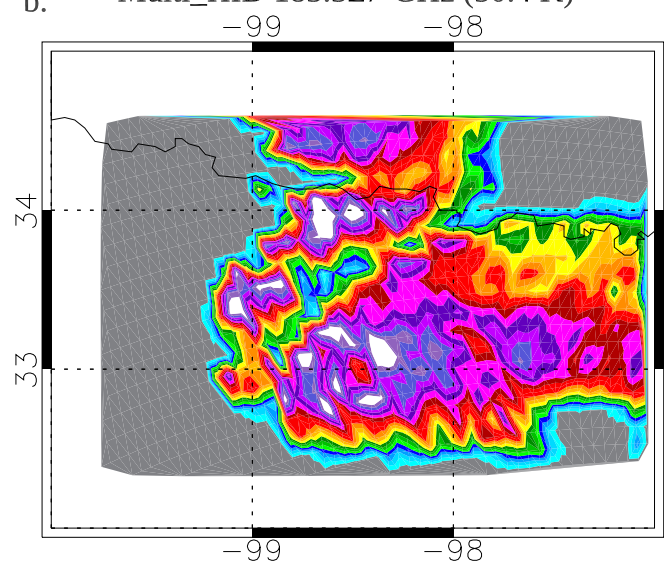

c. $\quad$ GMI $183.3 \pm 7-\mathrm{GHz}(85.9 \mathrm{~K})$

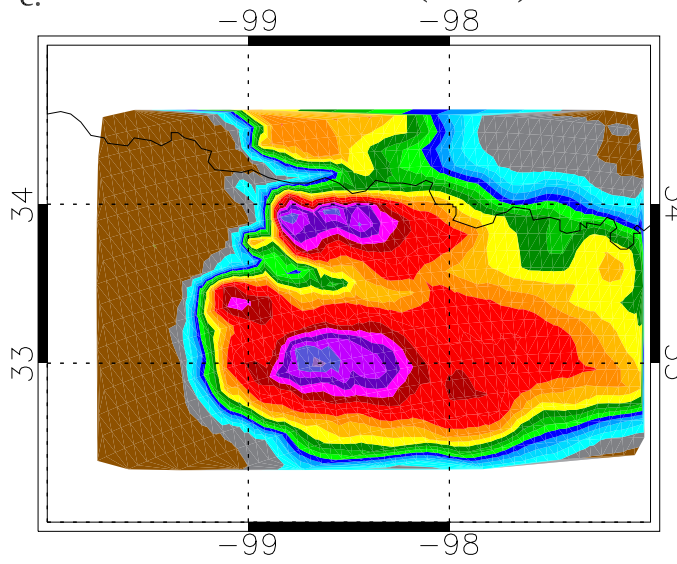

d. Single_HID $166.0-\mathrm{GHz}(35.9 \mathrm{~K})$

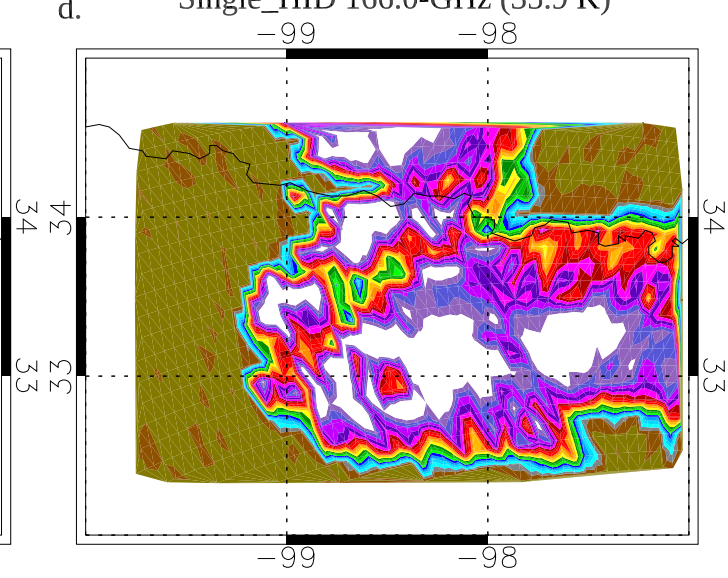

e. Multi_HID 166.0-GHz (54.9 K)

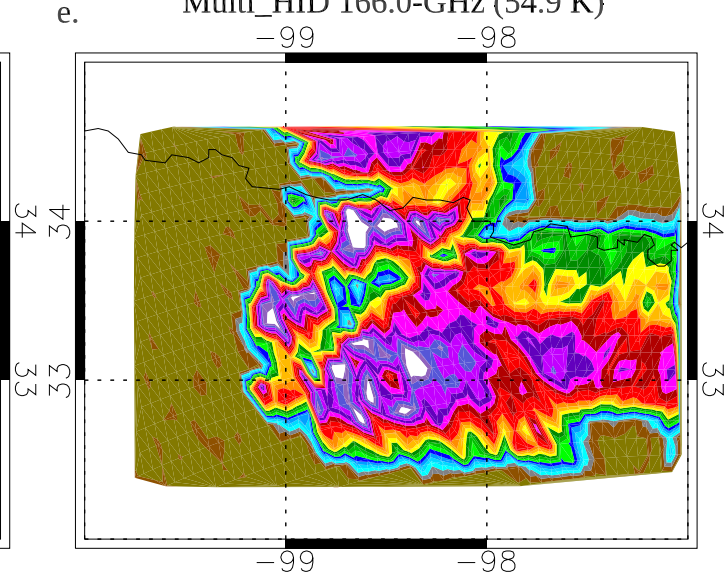

f.

GMI 166.0-GHz (82.5 K)

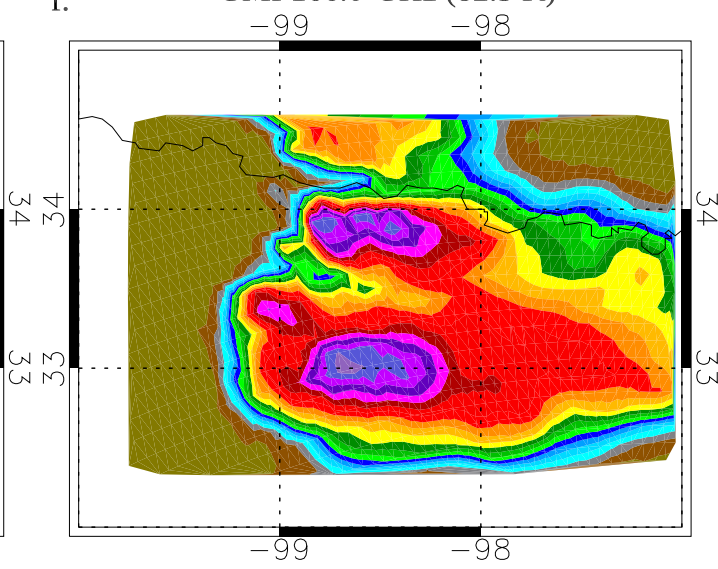

Brightness Temperature (K)

$\begin{array}{lllllllllllllllllllll}80 & 90 & 100 & 110 & 120 & 130 & 140 & 150 & 160 & 170 & 180 & 190 & 200 & 210 & 220 & 230 & 240 & 250 & 260 & 270 & 280\end{array}$

FIG. 2. The 183-GHz (a) simulated BTs where a single hydrometeor type was assigned to each radar grid box (Single_HID), (b) simulated BTs where multiple hydrometeor types were allowed at each grid box (Multi_HID) based on the HID beta function values (see text), and (c) observed BTs. (d)-(f) As in (a)-(c), but for 166 GHz. The minimum BT in each panel is given in the title of each panel. 
a. Single_HID $89.0-\mathrm{GHz}(97.2 \mathrm{~K})$

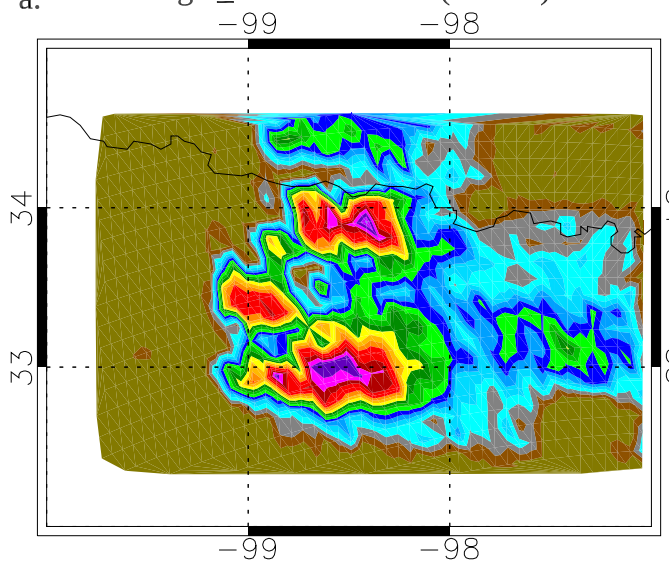

b. Multi_HID 89.0-GHz (93.0 K)
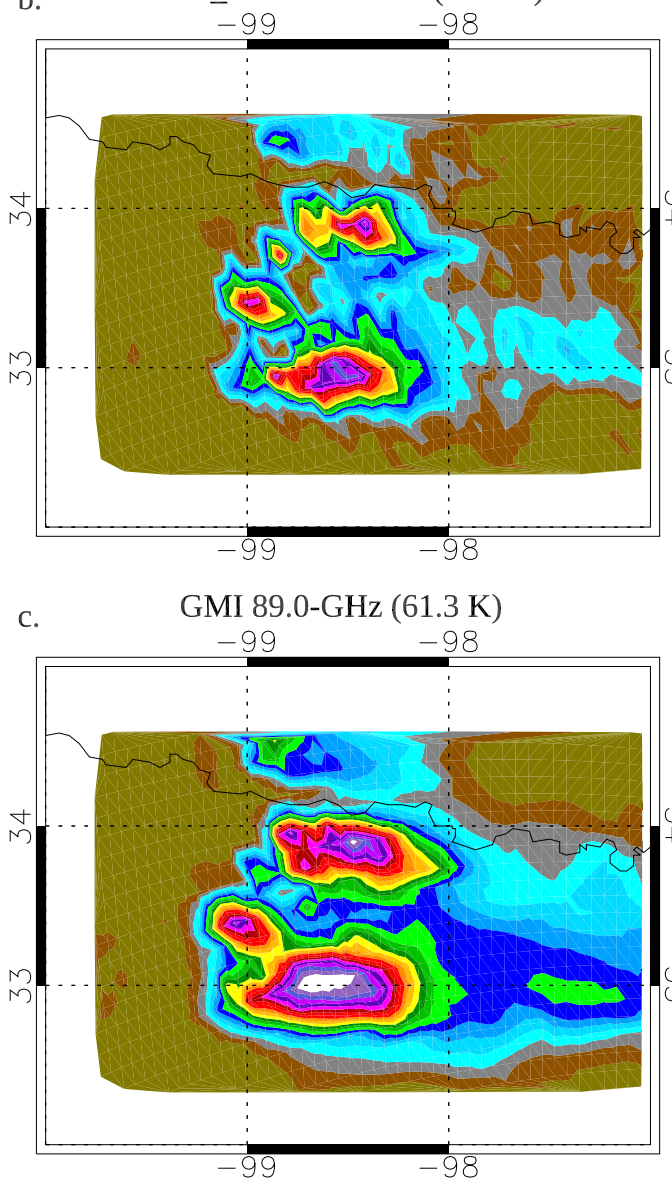

d. Single_HID 36.5-GHz (114.4 K)

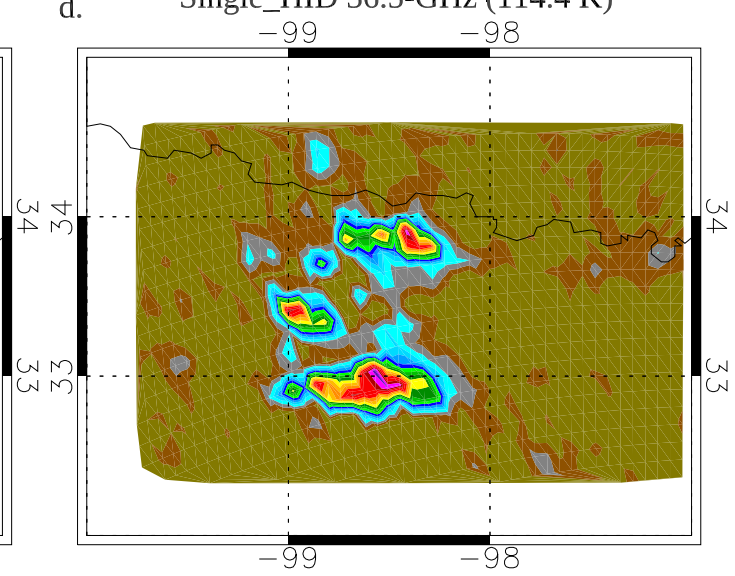

e. Multi_HID 36.5-GHz (126.1 K)

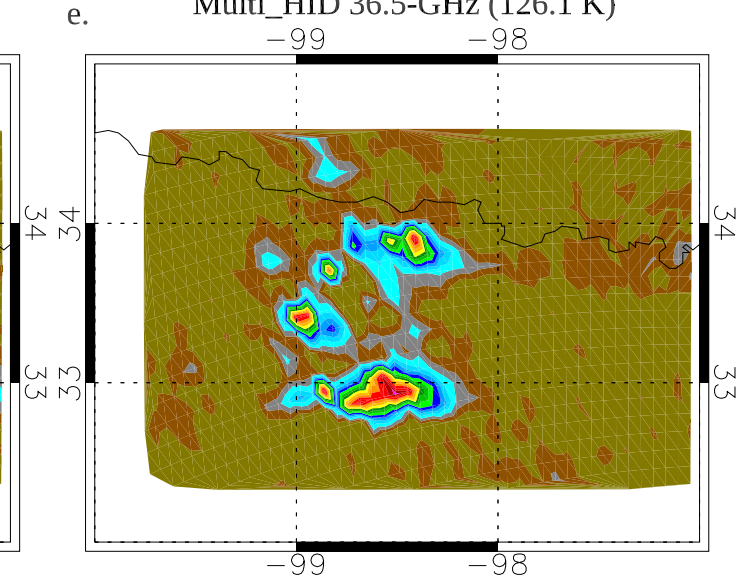

$\mathrm{f}$.

f. $\quad$ GMI $36.5-\mathrm{GHz}(87.0 \mathrm{~K})$

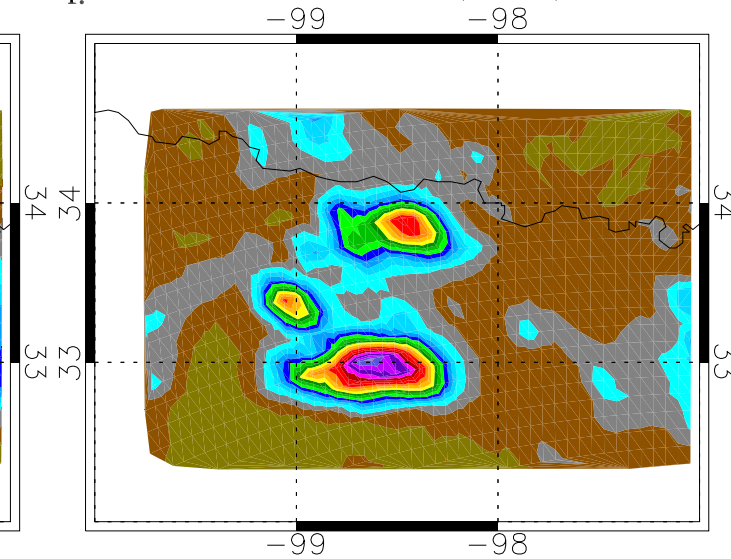

Brightness Temperature (K)

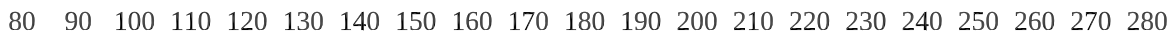
FIG. 3. As in Fig. 2, but for 89 and $36 \mathrm{GHz}$. 
a. Single_HID 18.7-GHz (160.5 K)

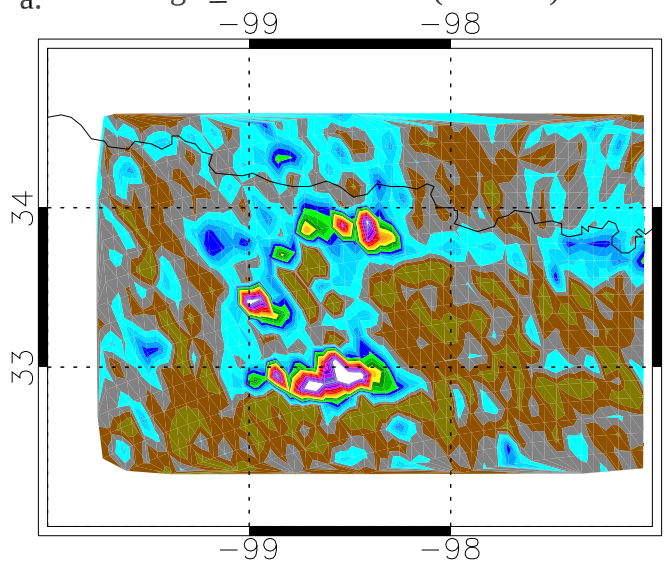

b. Multi_HID 18.7-GHz (182.3 K)
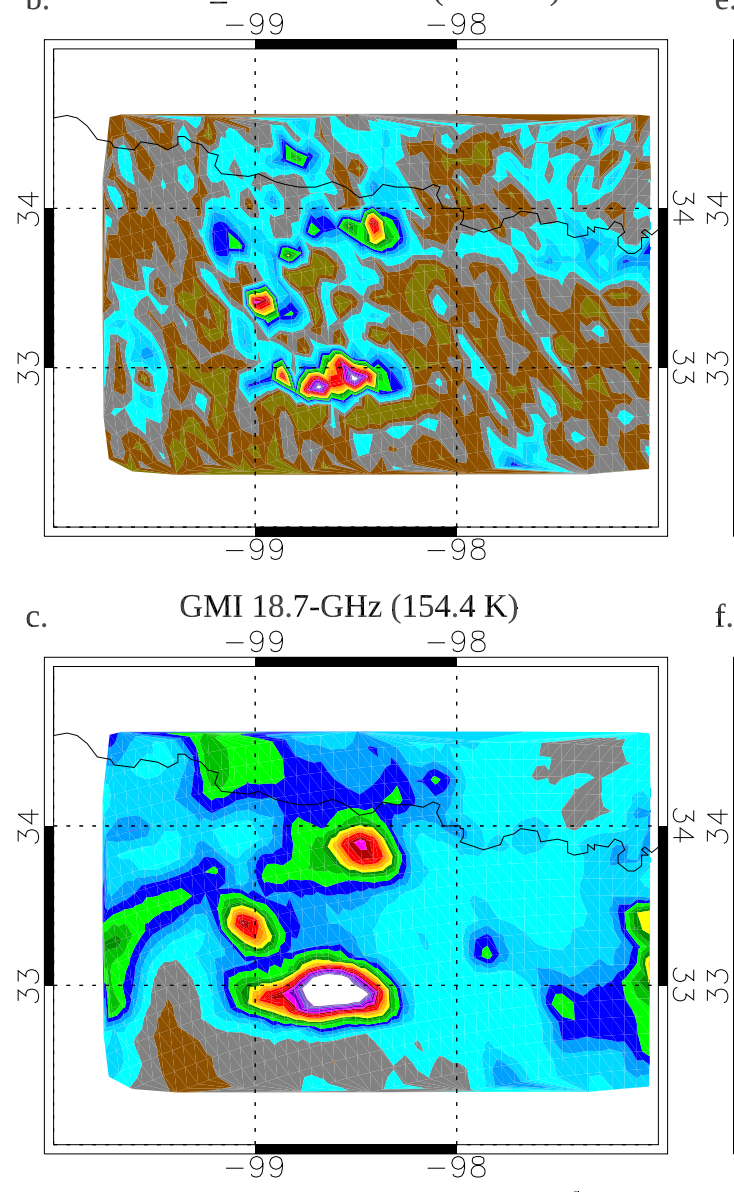

d. Single_HID 10.7-GHz (206.8 K)

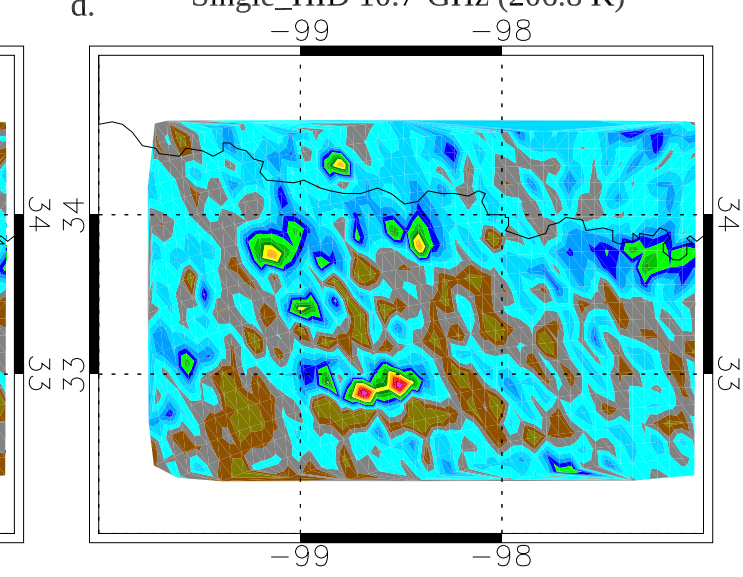

e. Multi_HID 10.7-GHz (229.6 K)

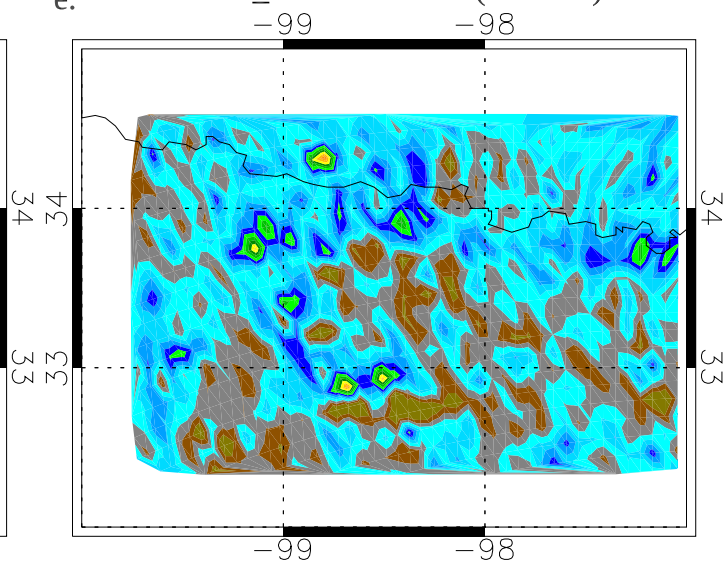

f.
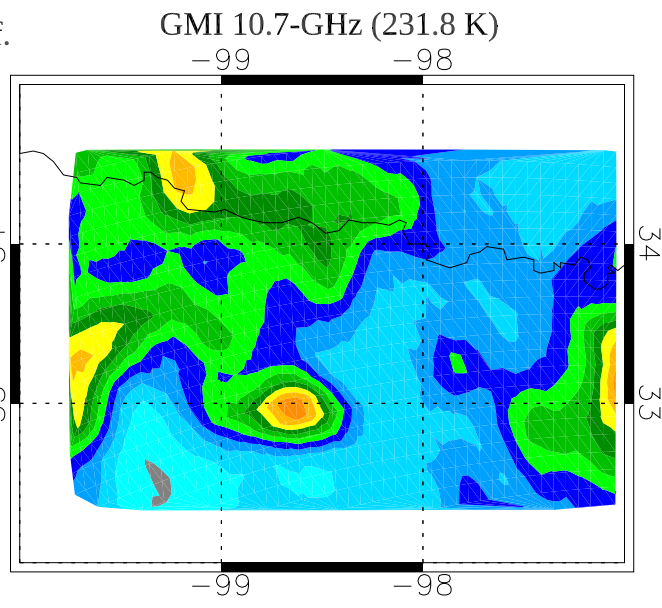

Brightness Temperature $(\mathrm{K})$

$190195200205210215220 \quad 225230235240245250255260 \quad 265270275280285290$ FIG. 4. As in Fig. 2, but for 18 and $10 \mathrm{GHz}$. 
of the reflectivity is assigned to hail $\left(6 \times 10^{4} \mathrm{~mm}^{6} \mathrm{~m}^{-3}\right.$ or $48 \mathrm{dBZ})$ and $4 \times 10^{4} \mathrm{~mm}^{6} \mathrm{~m}^{-3}(46 \mathrm{dBZ})$ is assigned to high-density graupel. Then PSDs were calculated as described above using the apportioned $Z_{h}$ values to calculate $N_{w}$ for each hydrometeor species that had a nonzero beta value at each grid point.

The Multi_HID simulation is identical to Single_HID except multiple hydrometeor types are allowed at each grid point. The results of the Multi_HID simulation are shown in the middle row of Figs. 2-4. The biggest change between the two simulations is observed at the two highest frequencies (Fig. 2). Allowing multiple hydrometeor species at each grid point substantially reduces the scattering in the anvil region, in much better agreement with the observations. Multi_HID also reduces BTs in the hail hole, in better agreement with observed values. BTs in the anvil region to the east also increased for the $89-\mathrm{GHz}$ frequency (Fig. 3) in going from Single_HID to Multi_HID. The Single_HID simulation had characterized the anvil well at $89 \mathrm{GHz}$, and Multi_HID degrades this performance. Changing from a single hydrometeor type to multiple types per grid box has relatively little impact on BTs at 10-36 GHz. However, the strongest scattering weakens at the two lowest frequencies leading to poorer agreement with minimum observed BTs at $18 \mathrm{GHz}$ and better agreement at $10 \mathrm{GHz}$.

Figure 5 shows the difference in vertically integrated mass of various hydrometeor species between the Multi_HID and Single_HID simulations. The values from Single_HID were subtracted from the corresponding Multi_HID values. There is a net reduction in mass for the Multi_HID simulation-the particle types and sizes assigned to each grid point are constrained by the observed S-band $Z_{h}$, not by mass. The mass of each hydrometeor species was calculated using the sizes and densities from Table 1 assuming that all types were spheres. As indicated by the aspect ratios different from 1 in Table 1, not all the hydrometeor types were assumed to be spheres for the scattering simulations. But, here we are more interested in the relative masses between the two simulations, not the absolute value of hydrometeor mass.

Allowing multiple hydrometeor types at each grid point as opposed to using a single type generally causes the mass of snow and low-density graupel to decrease slightly, while the mass of hail and cloud ice decrease by greater amounts. High-density graupel is the only species in Fig. 5 that shows a substantial increase in mass for some columns for Multi_HID. Grid points that were assigned as entirely hail in Single_HID tend to become a mixture of hail and high-density graupel in
Multi_HID, so some mass shifts from one category to the other. The decreased cloud ice in the anvil region in Multi_HID appears to be the cause for decreased simulated scattering (increased BT) for the high-frequency channels in Figs. 2 and 3. The conversion of some hail mass to high-density graupel appears to reduce the magnitude of hail hole in Fig. 2. Here, a convective core composed of more high-density graupel and less hail gives a lower simulated BT for the 183- and $166-\mathrm{GHz}$ channels, in better agreement with observations.

As a further assessment of the impact of allowing multiple hydrometeor types at a grid point, Fig. 6 shows scatterplots of simulated BTs (Single_HID and Multi_HID) as a function of observed BTs, and Table 4 shows statistics [correlation coefficient, bias, and rootmean-square error (RMSE)] from this comparison. The bias and RMSE of Multi_HID relative to GMI BTs at 183 and $166 \mathrm{GHz}$ are substantially lower relative to the corresponding values from Single_HID. The 183- and 166-GHz scatterplots (Figs. 6a,b) exhibit a set of nearly constant BTs of 260 and $280 \mathrm{~K}$, respectively, associated with relatively warm, but varied GMI BTs. These constant simulated values at 166 and $183 \mathrm{GHz}$ are clear-air pixels in the simulations. They may result from the assumed horizontally homogeneous model atmosphere, with the observations being sensitive to actual horizontal variations in atmospheric emission in the high-frequency channels. In addition, some of these pixels may occur where the S-band radar does not detect particles that affect the GMI measurements (whether due to radar sensitivity or the cone of silence near the radar location). In contrast to the two highest frequencies, the bias and RMSE are higher for Multi_HID relative to Single_HID (Table 4) at $89 \mathrm{GHz}$. Figure $6 \mathrm{c}$ clearly shows Multi_HID values shifted upward (positive bias) relative to Single_HID. Similar to what is shown in the maps of BTs in Figs. 3 and 4, allowing multiple hydrometeor types at a grid box has little systematic impact on the three lowest frequencies (Figs. 6d-f). Despite the slightly worse agreement with observed BTs at $89 \mathrm{GHz}$, all remaining simulations will allow multiple hydrometeor types at a grid point because this is more representative of the actual atmosphere and provides much better agreement with observed BTs at 166 and $183 \mathrm{GHz}$.

\section{Varying size distributions}

The next set of simulations involved increasing $D_{o}$ for each hydrometeor species separately while holding $\mu$ constant. As an illustration of the impact of increasing $D_{o}$ on the PSDs, Fig. 7 shows some example PSDs for cloud ice, high-density graupel, and hail from 
a.

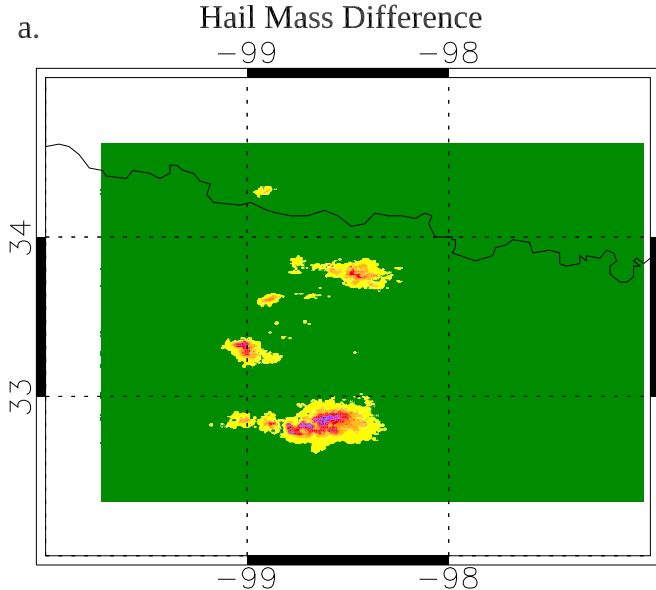

b.

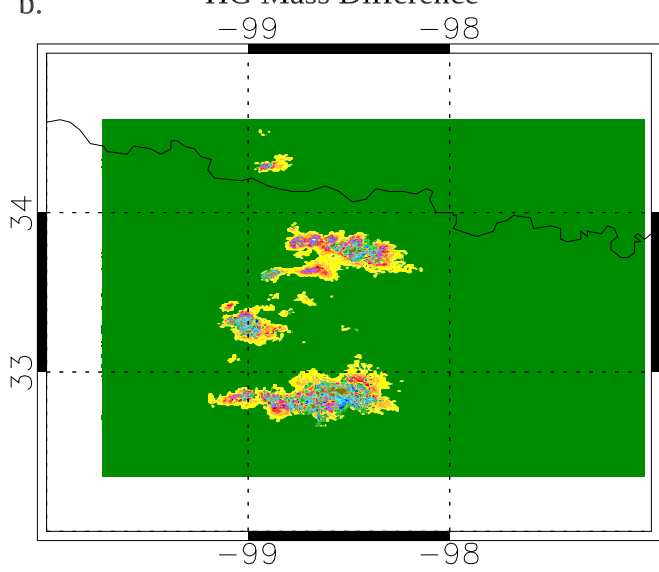

HG Mass Difference

G Mass Difference

c.

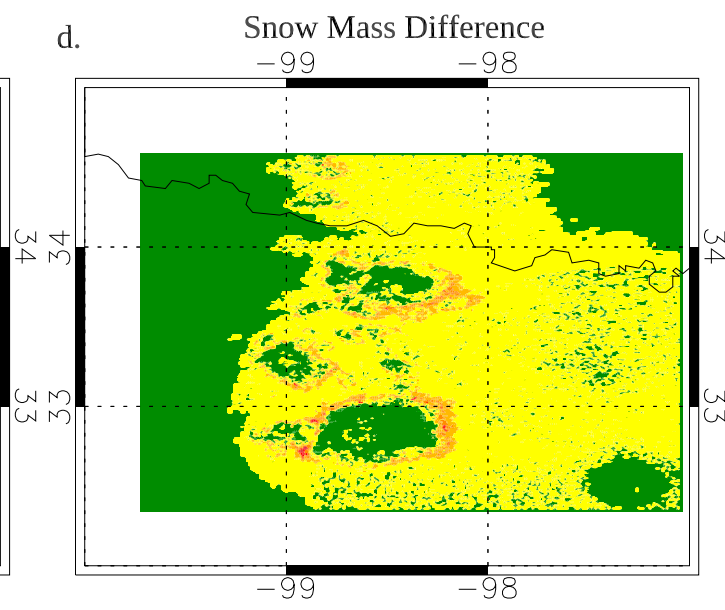

e.

Cloud Ice Mass Difference

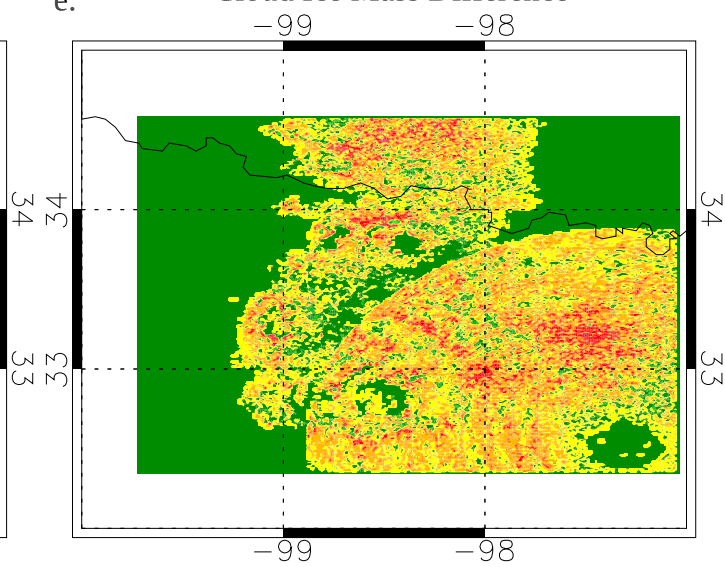

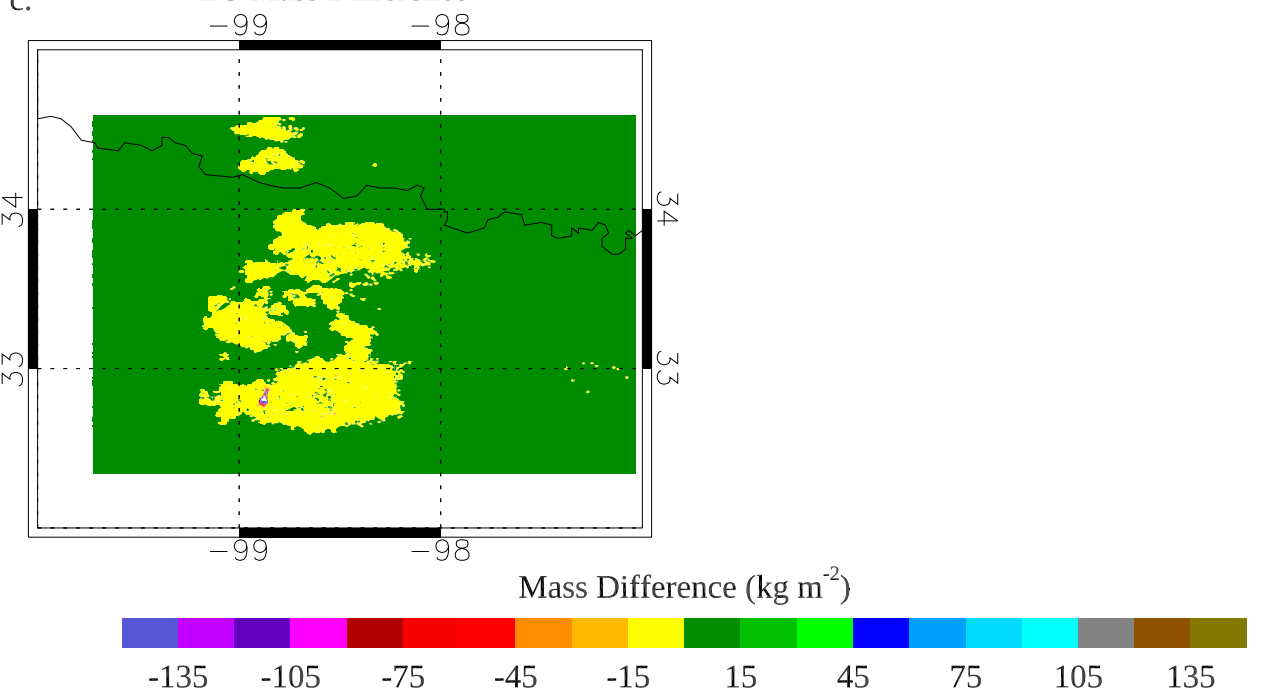

FIG. 5. The difference in vertically integrated (a) hail, (b) high-density graupel (HG), (c) low-density graupel (LG), (d) snow, and (e) cloud ice mass $\left(\mathrm{kg} \mathrm{m}^{-2}\right)$ between the Multi_HID and Single_HID (Multi_HID minus Single_HID) simulations. 
a.

$45 \quad 75105135165195225255285315$ GMI BT (K)

b.

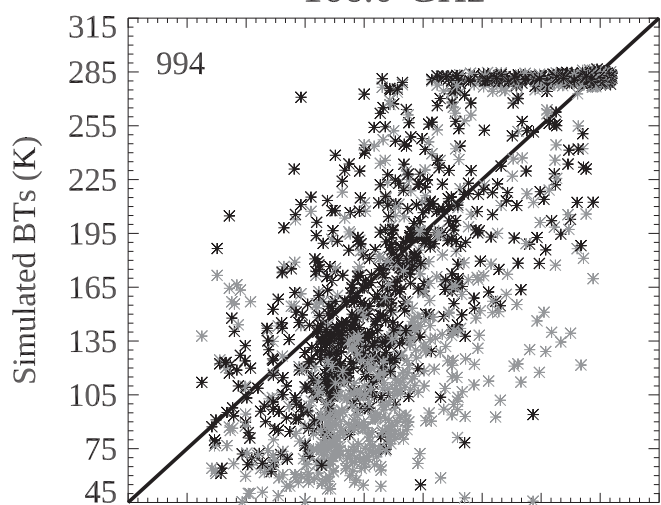

4575105135165195225255285315 GMI BT (K)

c.

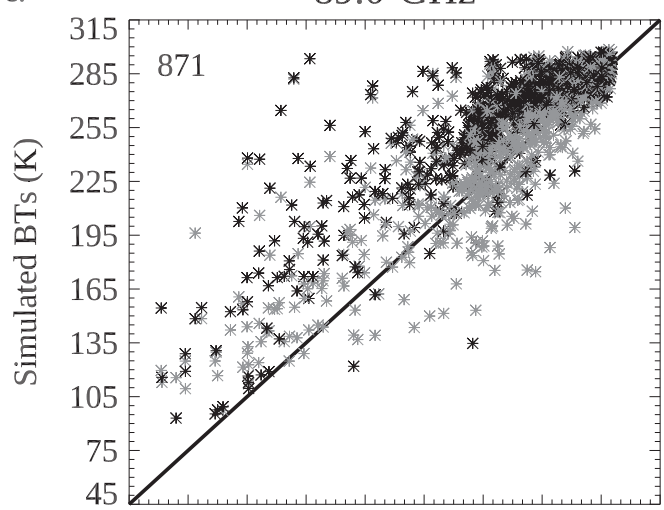

4575105135165195225255285315 GMI BT (K) d.

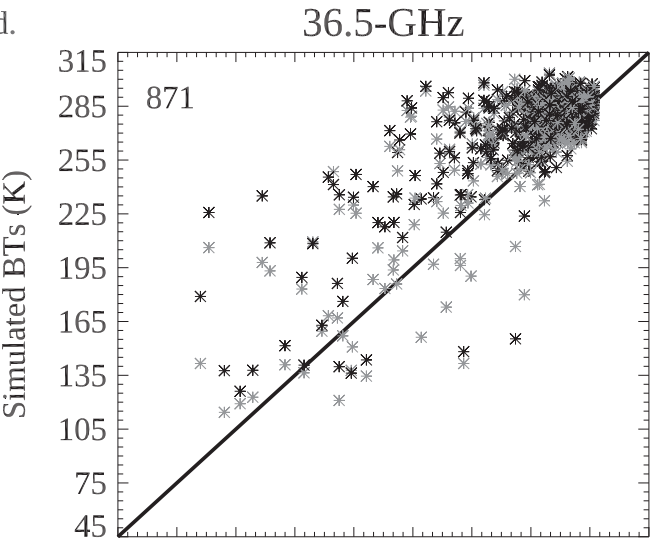

4575105135165195225255285315 GMI BT (K)

e.

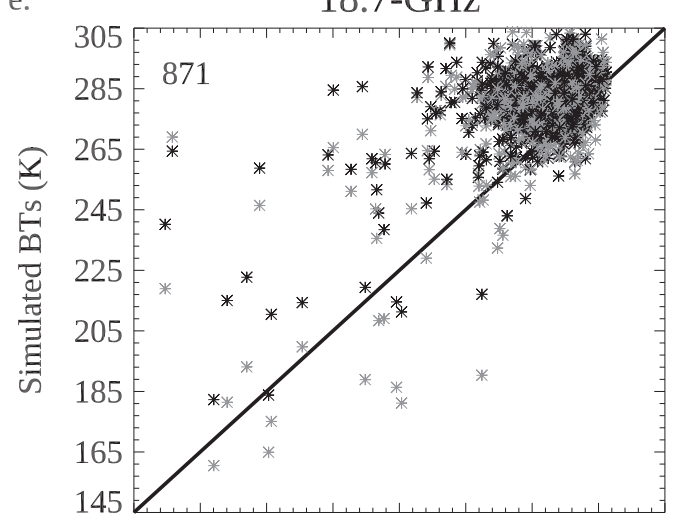

145165185205225245265285305

GMI BT (K)

f.

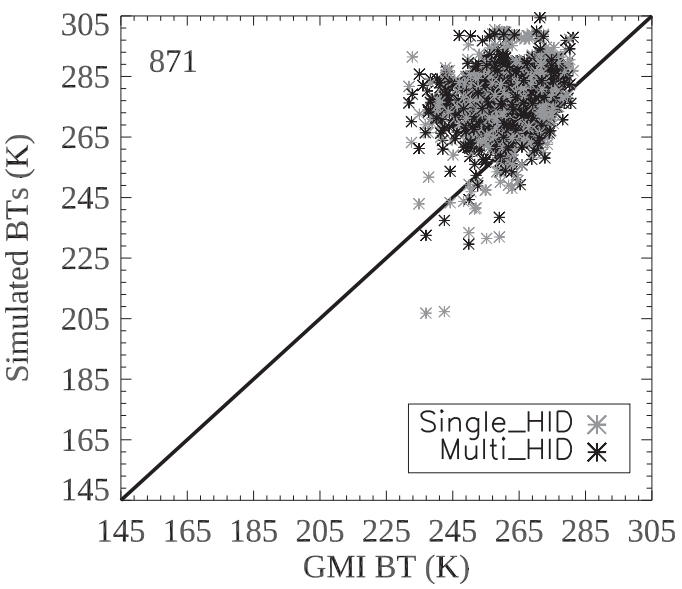

FIG. 6. Scatterplots showing simulated BTs as a function of observed BTs for (a) 183, (b) 166 (c) 89 , (d) 36 , (e) 18 , and (f) $10 \mathrm{GHz}$. The gray symbols correspond to a simulation where only one hydrometeor type was assigned to each radar grid box (Single_HID), while the black symbols correspond to a simulation where multiple hydrometeor types were allowed at each grid box (Multi_HID) as indicated by the key in (f). The number in the top-left corner of each panel is the sample size. 
TABLE 4. The correlation coefficient $r$, bias (K), and RMSE (K) for the comparison of Single_HID simulated BTs to the corresponding observed BTs as a function of frequency. These statistics are also given for the comparison between Multi_HID simulated and observed BTs.

\begin{tabular}{|c|c|c|c|c|c|c|}
\hline \multirow[b]{2}{*}{ Frequency $(\mathrm{GHz})$} & \multicolumn{3}{|c|}{ Single_HID } & \multicolumn{3}{|c|}{ Multi_HID } \\
\hline & $r$ & Bias & RMSE & $r$ & Bias & RMSE \\
\hline 183 & 0.84 & -32.33 & 58.78 & 0.85 & -9.33 & 36.54 \\
\hline 166 & 0.86 & -26.80 & 54.39 & 0.88 & -3.60 & 34.45 \\
\hline 89 & 0.88 & 7.42 & 23.30 & 0.87 & 19.46 & 30.75 \\
\hline 36 & 0.81 & 12.16 & 20.45 & 0.76 & 13.79 & 22.46 \\
\hline 18 & 0.62 & 11.43 & 17.42 & 0.56 & 12.31 & 17.92 \\
\hline 10 & 0.24 & 14.75 & 19.05 & 0.22 & 14.65 & 18.58 \\
\hline
\end{tabular}

various simulations. It is clear that increasing $D_{o}$ for any hydrometeor type substantially reduces the number concentration of the smallest particles. The increased number concentration of large particles is only obvious when plotted on a logarithmic axis as in Fig. 7. The increased $D_{o}$ yields vastly smaller total particle concentrations when summed across all size bins.

For Increase_CI_D0, $D_{o}$ of cloud ice was increased to $0.4 \mathrm{~mm}$ from its value of $0.2 \mathrm{~mm}$ for Multi_HID. Scatterplots depicting Increase_CI_D0 simulated BTs as a function of Multi_HID values are provided in Fig. 8. Maps of simulated BTs where $D_{o}$ of cloud ice is set to $0.4 \mathrm{~mm}$ are shown later in association with the discussion of the Adjust_Ice_D0 simulation. The 183- and $166-\mathrm{GHz}$ scatterplots show points that are generally shifted upward relative to the one-to-one line indicating less scattering (warmer BTs) when the cloud ice $D_{o}$ is increased. The bias of Increase_CI_D0 relative to Multi_HID at $183(166) \mathrm{GHz}$ is 9.76 (6.62) K. In contrast, points are clustered near the one-toone line for frequencies $\leq 89 \mathrm{GHz}$ (bias is negligible), suggesting that changing $D_{o}$ of cloud ice has little systematic impact on simulated BTs at lower frequencies.

The result that only the two highest frequencies show much sensitivity to changing the cloud ice PSD is consistent with several previous studies that have shown a greater sensitivity to frozen hydrometeor species with increasing frequency (e.g., Bennartz and Bauer 2003; Hong et al. 2005; Marra et al. 2017). Galligani et al. (2013) used ARTS and a monodisperse PSD to show that increased scattering occurs at 37 and $85 \mathrm{GHz}$ for larger ice hydrometeors. This seems to contradict results shown here where increasing $D_{o}$ of cloud ice leads to less scattering for frequencies $\geq 166 \mathrm{GHz}$. However, we use a normalized gamma distribution for the cloud ice PSD and a fixed $Z_{h}$. Bennartz and Petty (2001) suggest that under these conditions, increasing

Sample Particle Size Distributions

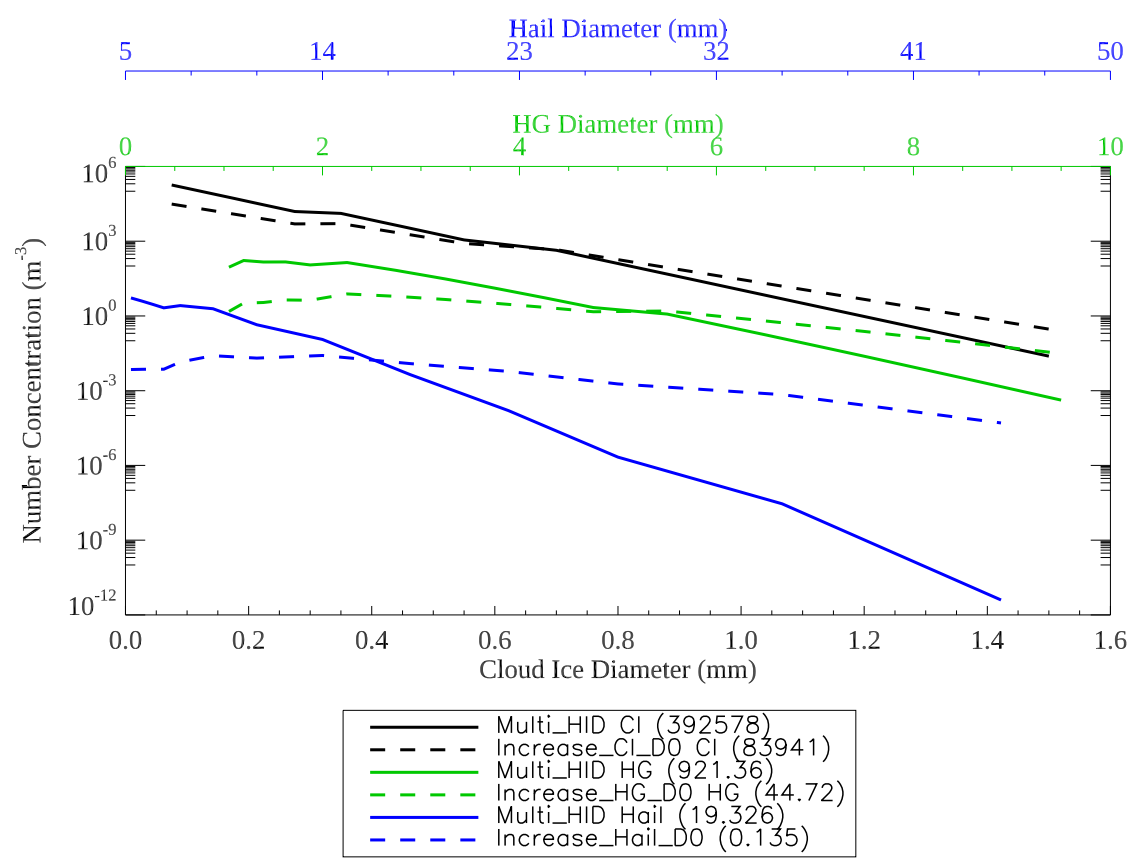

FIG. 7. Sample PSDs for cloud ice (CI; black), high-density graupel (HG; green), and hail (blue) from the control simulation (Multi_HID) and simulations where $D_{o}$ was increased for each hydrometeor type. The total particle concentration $\left(\mathrm{m}^{-3}\right)$ summed across all size bins is listed in the legend at bottom. 
a.

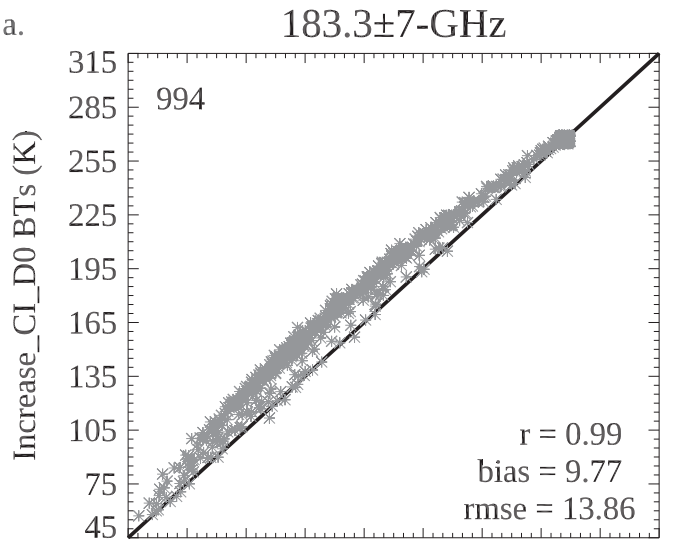

4575105135165195225255285315 Multi_HID BTs (K)

b.

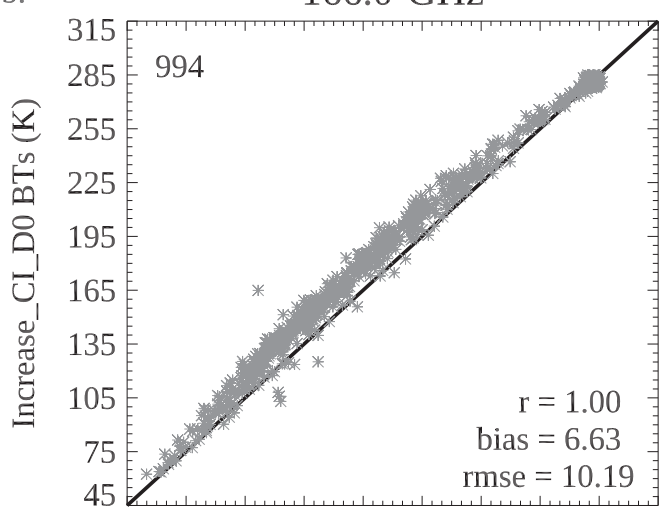

4575105135165195225255285315 Multi_HID BTs (K)

c.

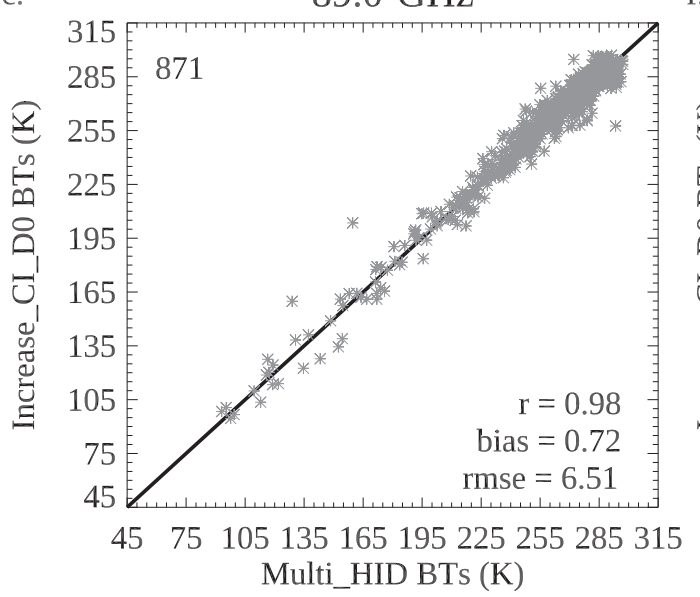

d.

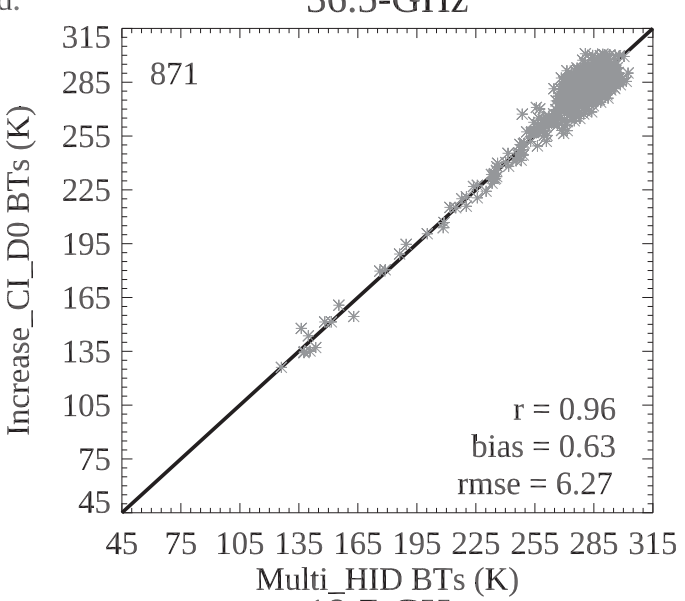

e.

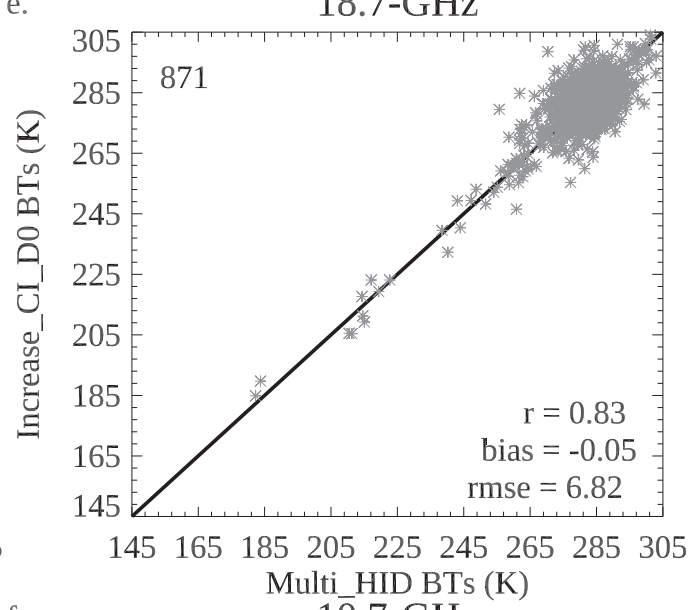

$f$.

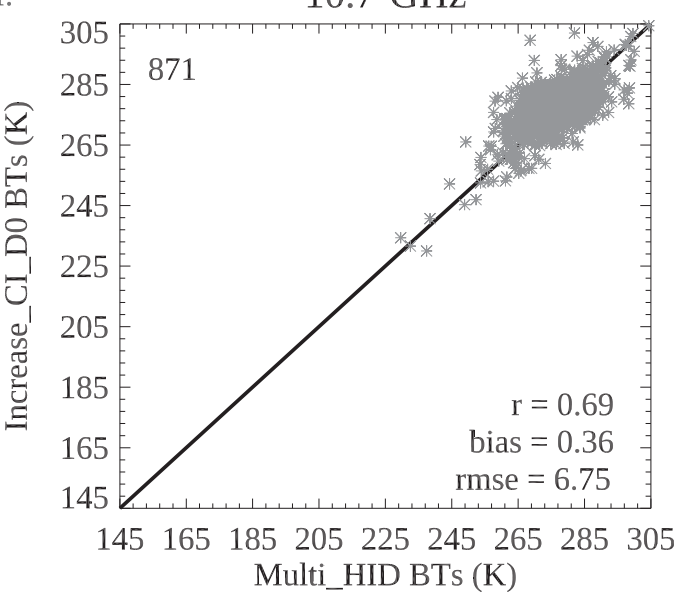

FIG. 8. Scatterplot of simulated BTs from Increase_CI_D0 $\left(D_{o}=0.4 \mathrm{~mm}\right.$ for cloud ice $)$ as a function of simulated BTs from Multi_HID ( $D_{o}=0.2 \mathrm{~mm}$ for cloud ice) valid at (a) 183 , (b) 166 (c) 89 , (d) 36 , (e) 18 , and (f) $10 \mathrm{GHz}$. The correlation coefficient $r$, bias $(\mathrm{K})$, and RMSE $(\mathrm{K})$ are given in the bottom-right corner of each panel. Sample size is given in the top-left corner of each panel. 

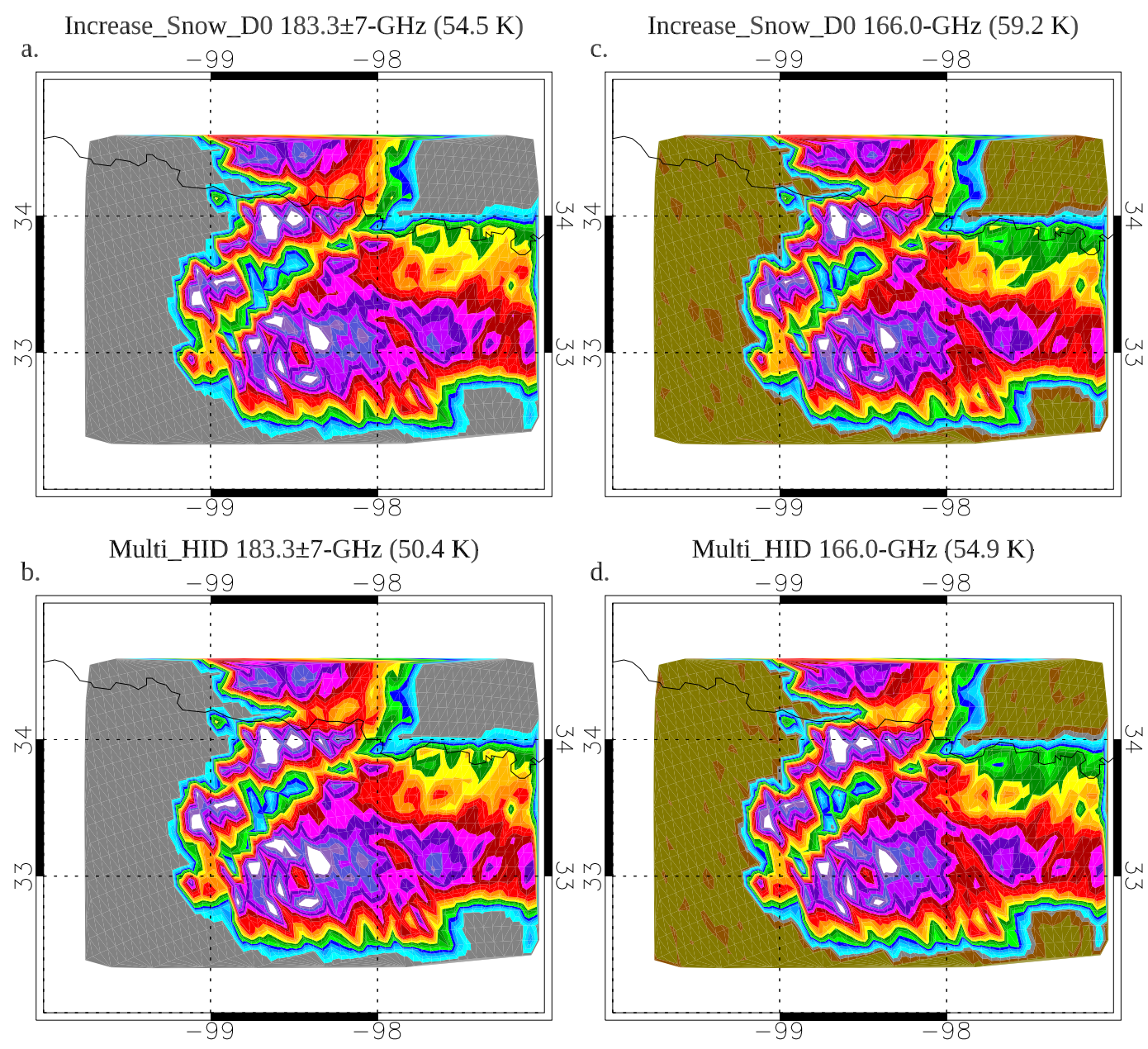

Brightness Temperature (K)

$\begin{array}{lllllllllllllllllllll}80 & 90 & 100 & 110 & 120 & 130 & 140 & 150 & 160 & 170 & 180 & 190 & 200 & 210 & 220 & 230 & 240 & 250 & 260 & 270 & 280\end{array}$

FIG. 9. The 183-GHz (a) simulated BTs where $D_{o}=2.0 \mathrm{~mm}$ for snow (Increase_Snow_D0) and (b) simulated BTs from the control simulation (Multi_HID; snow $D_{o}=0.5 \mathrm{~mm}$ ). (c),(d) As in (a) and (b), but for $166 \mathrm{GHz}$.

the size of particles actually leads to less scattering. For example, the bottom row of their Fig. 2 shows that for a given $Z_{h}$, the volume extinction coefficient increases for decreasing size (increasing size ratio). Under conditions of fixed $Z_{h}$, increasing $D_{o}$ results in smaller number concentrations (e.g., Fig. 7), which may be contributing to the increase in BT at the highest frequencies. In particular, the smaller number of crystals resulting from the increase in $D_{o}$ presents a smaller surface area, resulting in less scattering (Hartmann 1994).

Bennartz and Bauer (2003) found the sensitivity to snow increases with frequency. In addition, You et al. (2017) found poor snow detection capability for GMI channels $<89 \mathrm{GHz}$. Hence, it is not surprising that maps of simulated BTs for Increase_Snow_D0 (not shown) where the snow $D_{o}$ was increased to $2.0 \mathrm{~mm}$ look nearly identical to those of Multi_HID (0.5 mm; Table 3) shown for frequencies $\leq 89 \mathrm{GHz}$ in Figs. 3 and 4 . However, maps of BTs for both simulations at 183 and $166 \mathrm{GHz}$ (Fig. 9) are also nearly identical to one another, in contrast to the results of changing the PSD of cloud ice. Scatterplots of Increase_Snow_D0 BTs as a function of Multi_HID BTs shown in Fig. 10 confirm that these simulations appear to be insensitive to changes in the snow PSD with all the points clustered near the one-to-one line for every frequency. The bias between Increase_Snow_D0 and Multi_HID is $<1.0 \mathrm{~K}$, and the correlation coefficient is $>0.84$ for all frequencies except $10 \mathrm{GHz}$. Results of sensitivity simulations with a single hydrometeor species (not shown) suggest that the scattering effect of cloud ice (high density) at 166 and $183 \mathrm{GHz}$ is much stronger than that of snow (low 
a.

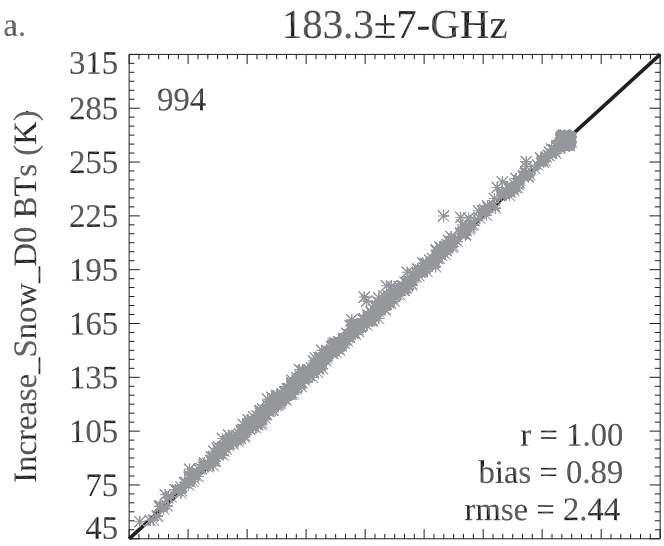

4575105135165195225255285315 Multi_HID BTs (K)

b.

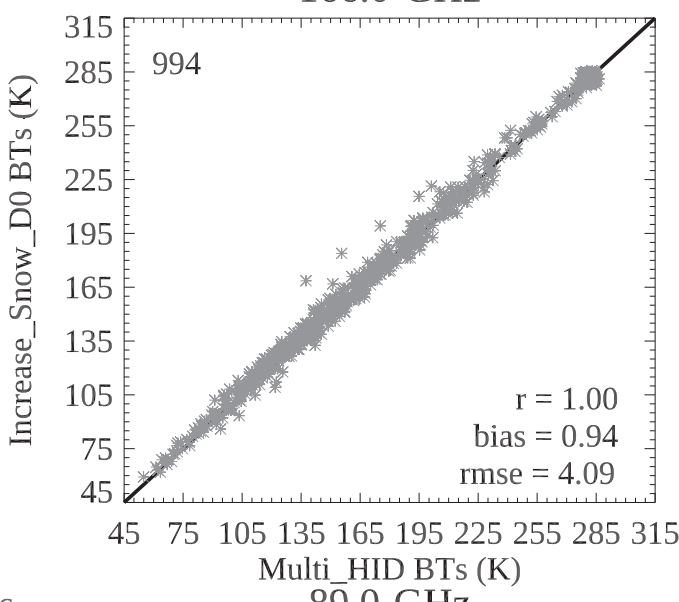

c.

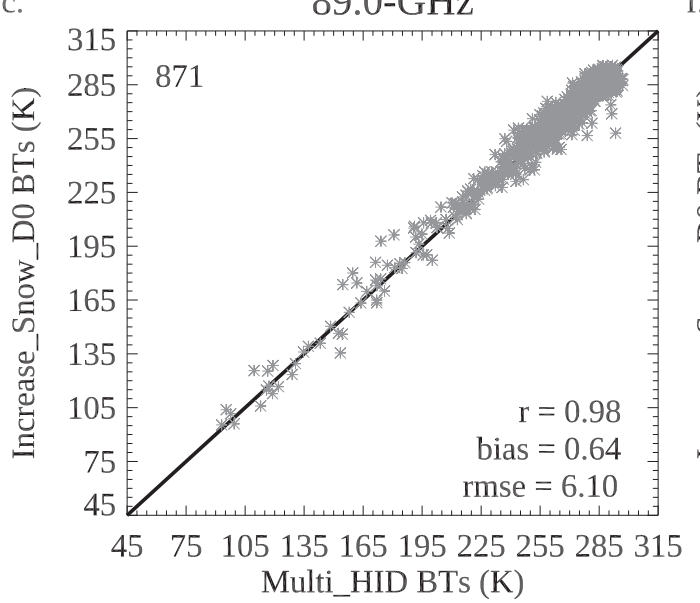

d.

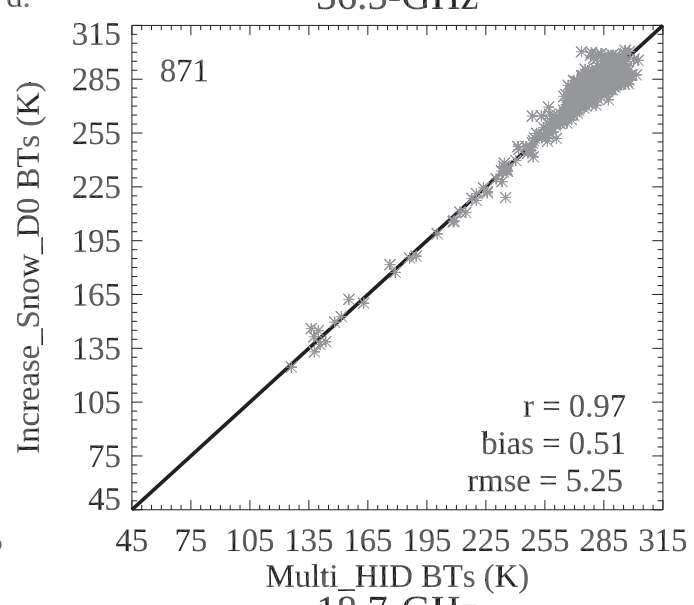

e.

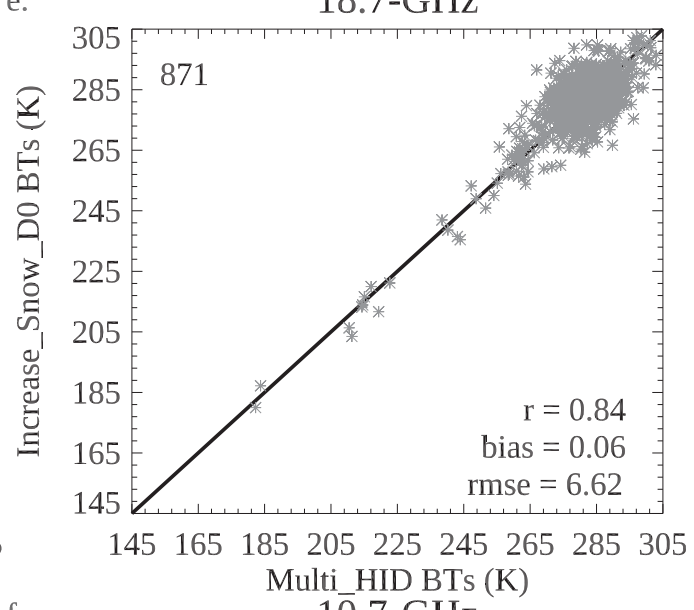

f.

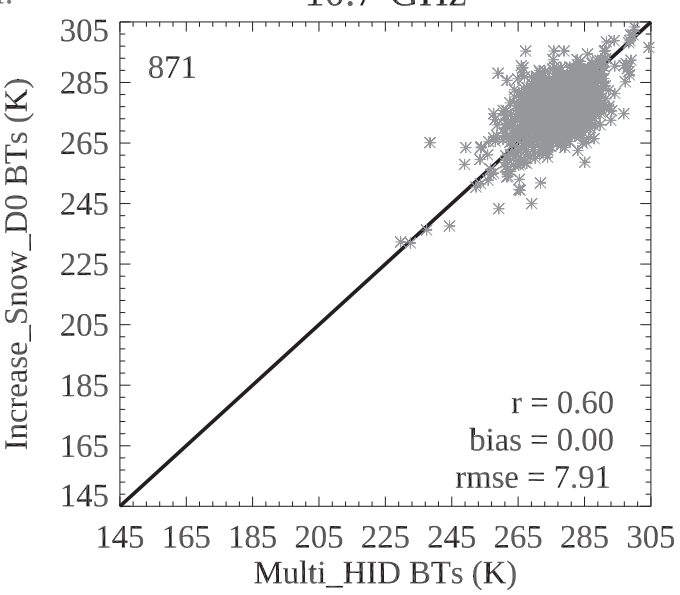

FIG. 10. As in Fig. 8, but showing Increase_Snow_D0 $\left(D_{o}=2.0 \mathrm{~mm}\right.$ for snow $)$ simulated BTs as a function of Multi_HID $\left(D_{o}=0.5 \mathrm{~mm}\right.$ for snow $)$ BTs.

density), consistent with Hong et al. (2005). Because all of the GMI pixels that sample snow also sample cloud ice, the impact of changing the snow PSD may be masked by the stronger influence of other hydrometeor species.
The number of GMI pixels that sample high-density graupel and hail are relatively small compared to the full number of pixels. Hence, maps of simulated BTs where parameters of the high-density graupel or hail PSDs are changed show little change from the control simulation 
and, thus, are not shown. To isolate the impact of changing these PSD parameters, scatterplots are presented that are limited to only those pixels that sample high-density graupel or hail. For example, Fig. 11 shows scatterplots of Increase_HG_D0 (high-density graupel $D_{o}=4.5 \mathrm{~mm}$ ) simulated BTs as a function of Multi_HID BTs (high-density graupel $D_{o}=2.5 \mathrm{~mm}$; Table 3 ). Note the sample size given in the top-left corner of each panel is different for each frequency because of the different spatial resolution of each channel. Similar to what was observed for cloud ice, shifting $D_{o}$ to a larger value for high-density graupel causes simulated BTs to warm. In other words, increasing $D_{o}$ (thereby decreasing particle number concentration) under conditions of fixed $Z_{h}$ results in a weaker scattering signature from highdensity graupel. However, in contrast to ice crystals, BTs at frequencies as low as $36 \mathrm{GHz}$ are noticeably impacted by the change in high-density graupel PSD. Using higher-resolution airborne passive microwave data, Leppert and Cecil (2015) showed that frequencies $\geq 36 \mathrm{GHz}$ are sensitive to the presence of graupel. The statistics presented in Fig. 11 indicate that relative to Multi_HID, Increase_HG_D0 BTs are biased high by $>5 \mathrm{~K}$ for frequencies $\geq 36 \mathrm{GHz}$, whereas the bias is $<1 \mathrm{~K}$ for frequencies $\leq 18 \mathrm{GHz}$. In particular, the $89-\mathrm{GHz}$ channel shows the biggest change as a result of changing the high-density graupel PSD with a bias (RMSE) of $13.4(26.0) \mathrm{K}$. A simulation where $D_{o}$ for low-density graupel was increased relative to that of the control simulation (not shown) showed results similar to that of high-density graupel except that BTs increased with increasing $D_{o}$ at frequencies $\geq 89 \mathrm{GHz}$, but not at $36 \mathrm{GHz}$.

Similar to Fig. 11, Fig. 12 shows the impact of increasing hail $D_{o}$ from the Multi_HID value of $6.0 \mathrm{~mm}$ to the Increase_Hail_D0 value of $18.0 \mathrm{~mm}$ using only pixels where hail was sampled. Results from Leppert and Cecil (2015) suggest a scattering signature from hail at all frequencies examined from 10 to $183 \mathrm{GHz}$. Ferraro et al. (2015) used channels between 89 and $183 \mathrm{GHz}$ for hail detection, and numerous other hail detection/climatology studies have exploited the sensitivity of frequencies near 19,37, and/or $85 \mathrm{GHz}$ to the presence of hail (Cecil 2009; Cecil and Blankenship 2012; Mroz et al. 2017; Ni et al. 2017). Thus, it is somewhat surprising that all frequencies shown in Fig. 12 suggest relatively little sensitivity to changing the PSD of hail. However, the data points with the lowest BT for Multi_HID tend to have increased BT from Increase_Hail_D0 for frequencies $\leq 36 \mathrm{GHz}$. This behavior is consistent with the response of higherfrequency channels to other hydrometeor species. Two other simulations were carried out with hail $D_{o}$ of
10.0 and $14.0 \mathrm{~mm}$, and results from these were similar to what is shown for Increase_Hail_D0.

One possible explanation for the apparent lack of sensitivity to hail for the highest frequencies is due to Mie effects on the single-scattering properties of hail (e.g., Casella et al. 2008; Mroz et al. 2017). In particular, Mroz et al. (2017) show that the single-scattering albedo for hail increases with size up to a point and then decreases with further increases in size at higher frequencies (cf. their Fig. 6a). The hail size at which the single-scattering albedo peaks decreases with increasing frequency. Thus, at higher frequencies, relatively small hailstones can be in a regime where their effective scattering decreases rapidly with frequency. This may at least partly explain why frequencies $\geq 89 \mathrm{GHz}$ appear insensitive to changes in the hail PSD (Fig. 12). Observed correlations between low BT at the high frequencies and hail occurrence (e.g., Ferraro et al. 2015) may result as a coincidence of storms with hail also tending to have large concentrations of graupel elsewhere within the storm.

Another explanation for the general lack of a clear response to changes in the hail PSD observed here, except for a handful of pixels at 18 and $36 \mathrm{GHz}$ may be related to nonuniform beamfilling. The area within a simulated satellite pixel covered by hail may be small enough that the simulated BT over the pixel is relatively insensitive to the hail. In an attempt to test this, the percentage of model grid points contained within the satellite line of sight that contained hail (i.e., beta value for hail $>0$ ) was calculated. Then separate scatterplots were created similar to Fig. 12 except that one set of plots showed pixels that contained a percentage of hail greater than the median percentage (the median percentage ranges from $3.5 \%$ at $10 \mathrm{GHz}$ to $13.8 \%$ at $183 \mathrm{GHz}$ ), while the other set of plots contained pixels with hail coverage below the median value. These scatterplots (not shown) indicate that the pixels at 18 and $36 \mathrm{GHz}$ that occur substantially above the one-to-one line (i.e., show greatest sensitivity to changing hail PSD) contain a relatively large coverage by hail. This simple test suggests that a greater sensitivity to hail and associated changes in its PSD may be observed if hail filled the volume of GMI's line of sight better.

\section{Extreme hail size versus extreme concentrations}

To address the third question listed in the introduction (i.e., how low can BTs reach given realistic but extreme distributions of hail), simulations were conducted with only $0.5-$ or $20-\mathrm{cm}$ diameter hailstones. By definition, $0.5 \mathrm{~cm}$ is the size that distinguishes hail from graupel 
a.

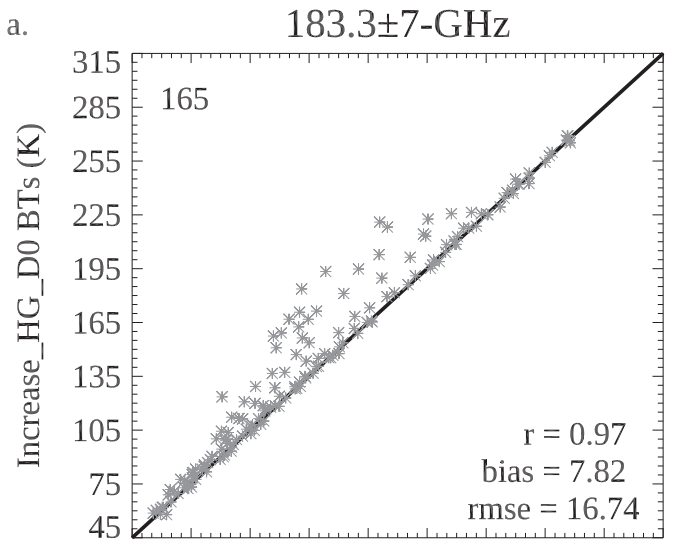

4575105135165195225255285315 Multi_HID BTs (K)

b.

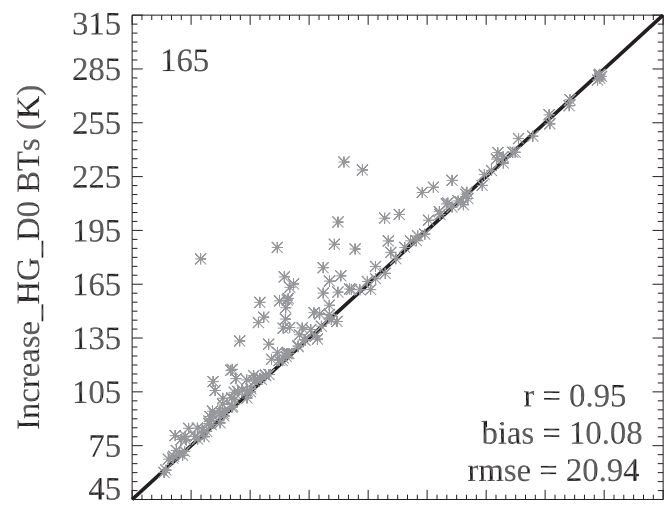

4575105135165195225255285315 Multi_HID BTs (K)

c.

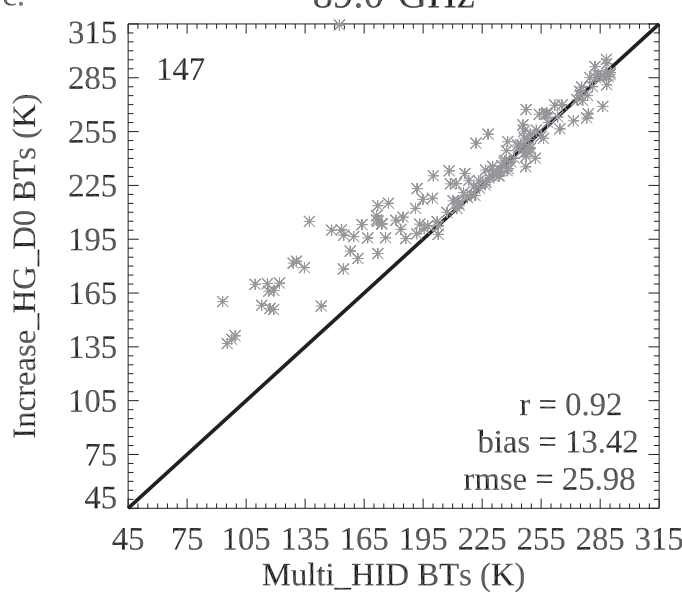

d.

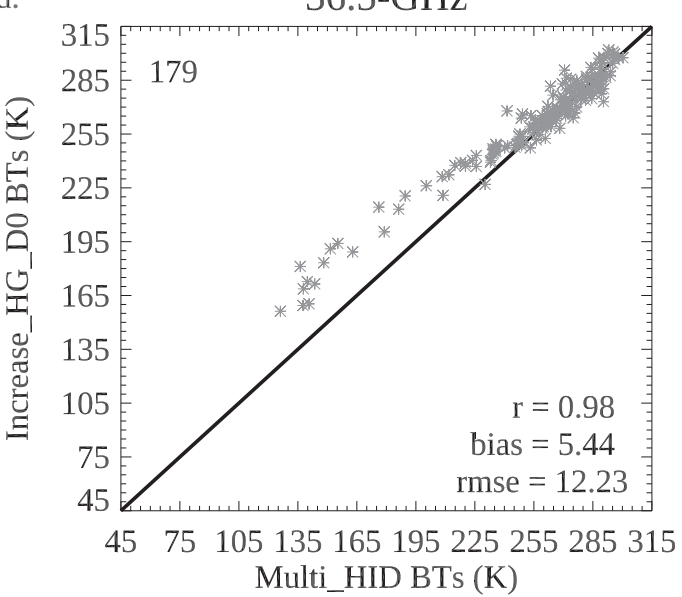

e.

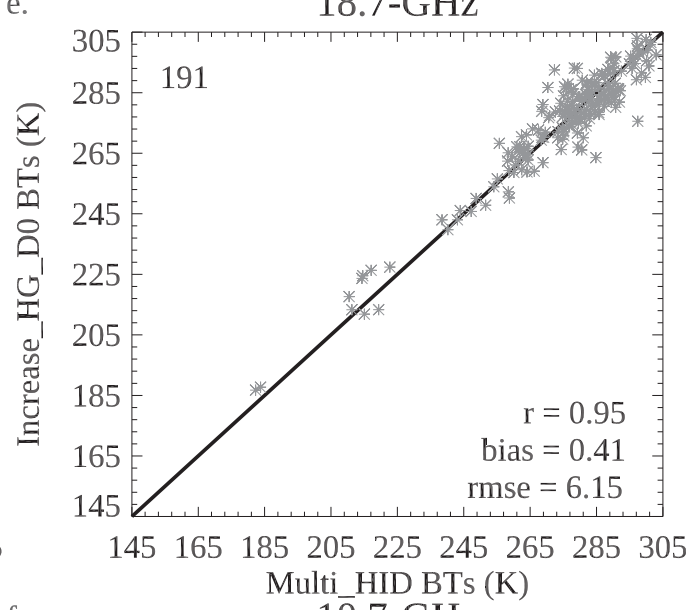

f.

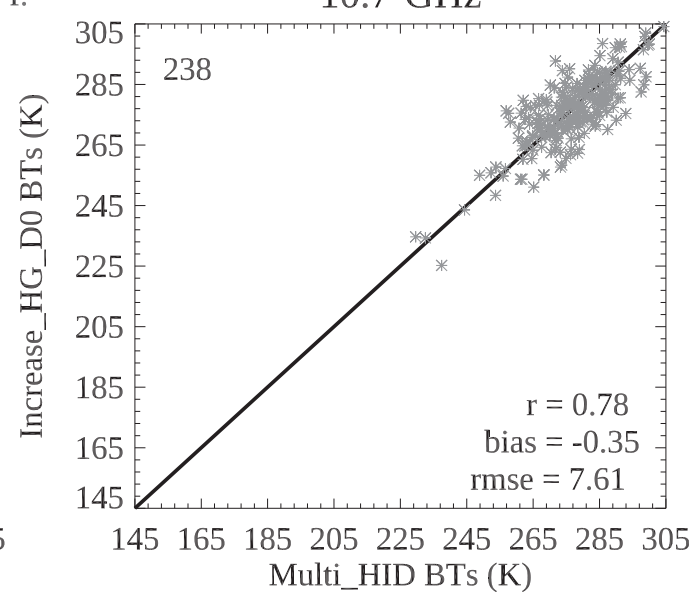

FIG. 11. Scatterplot of simulated BTs from Increase_HG_D0 $\left(D_{o}=4.5 \mathrm{~mm}\right.$ for high-density graupel $)$ as a function of simulated BTs from Multi_HID $\left(D_{o}=2.5 \mathrm{~mm}\right.$ for high-density graupel) valid at (a) 183 , (b) 166 (c) 89 , (d) 36, (e) 18, and (f) $10 \mathrm{GHz}$. Only pixels that sample high-density graupel are included here (sample size is given in the top-left corner of each panel). The correlation coefficient $r$, bias (K), and RMSE (K) are given in the bottomright corner of each panel. 
a.

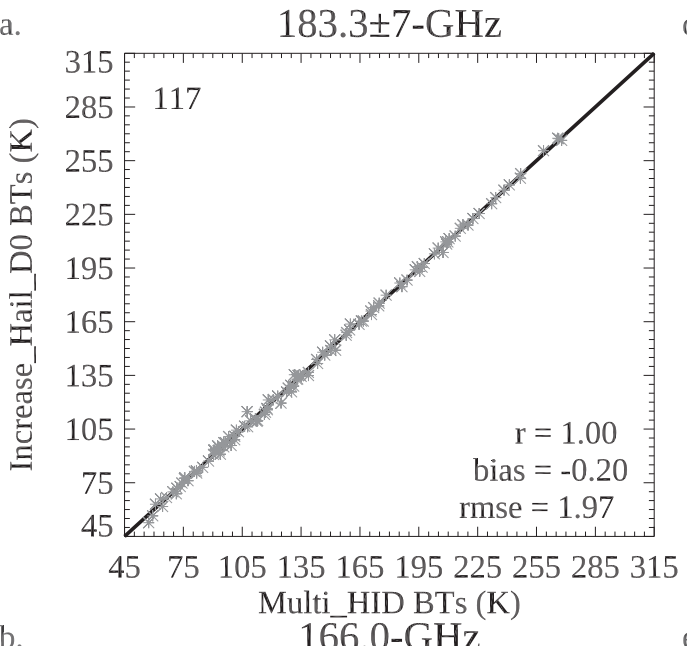

b.

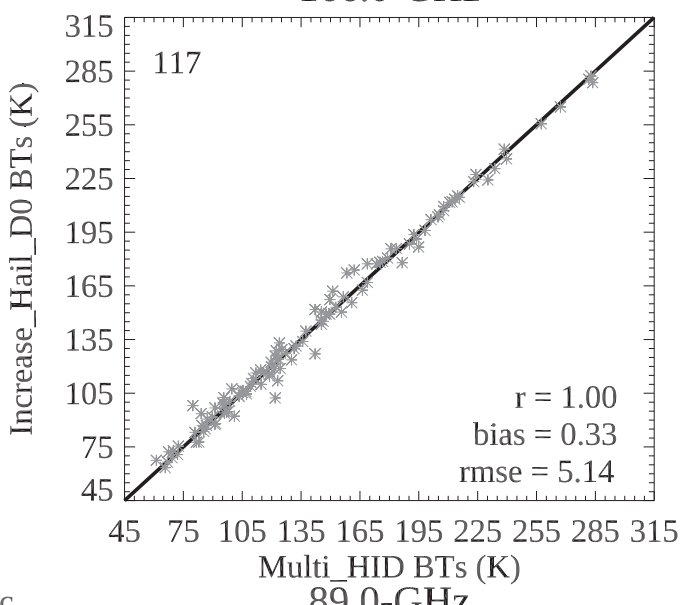

c.

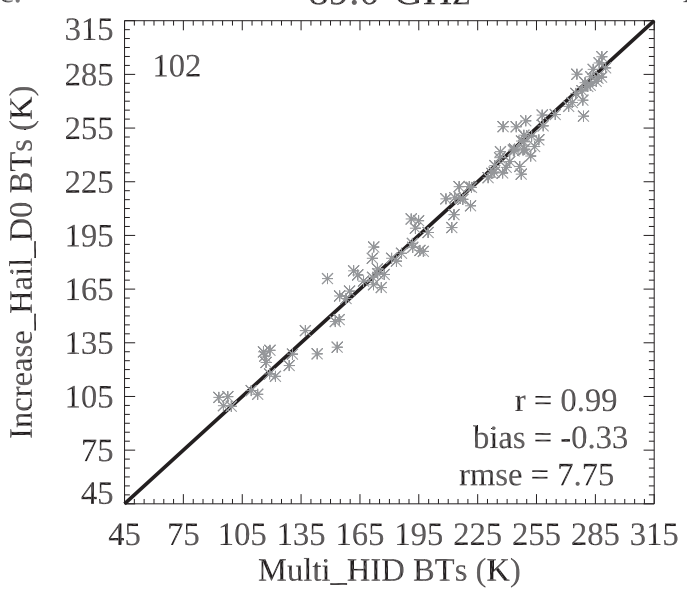

d.

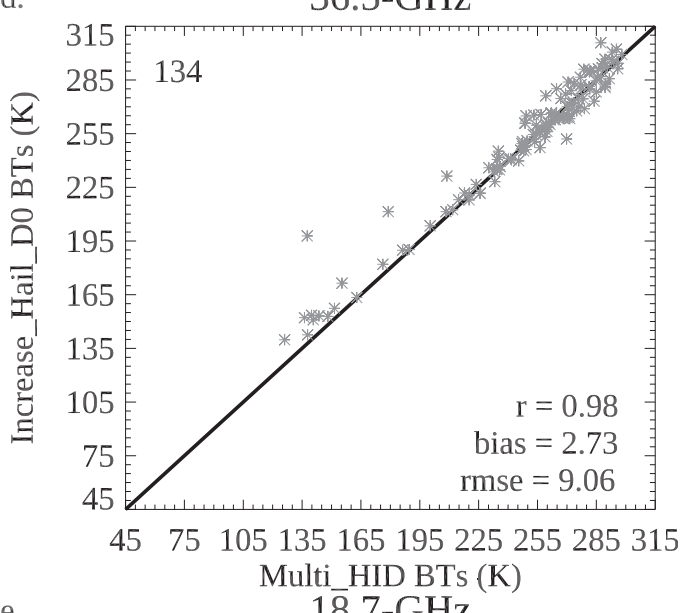

e.
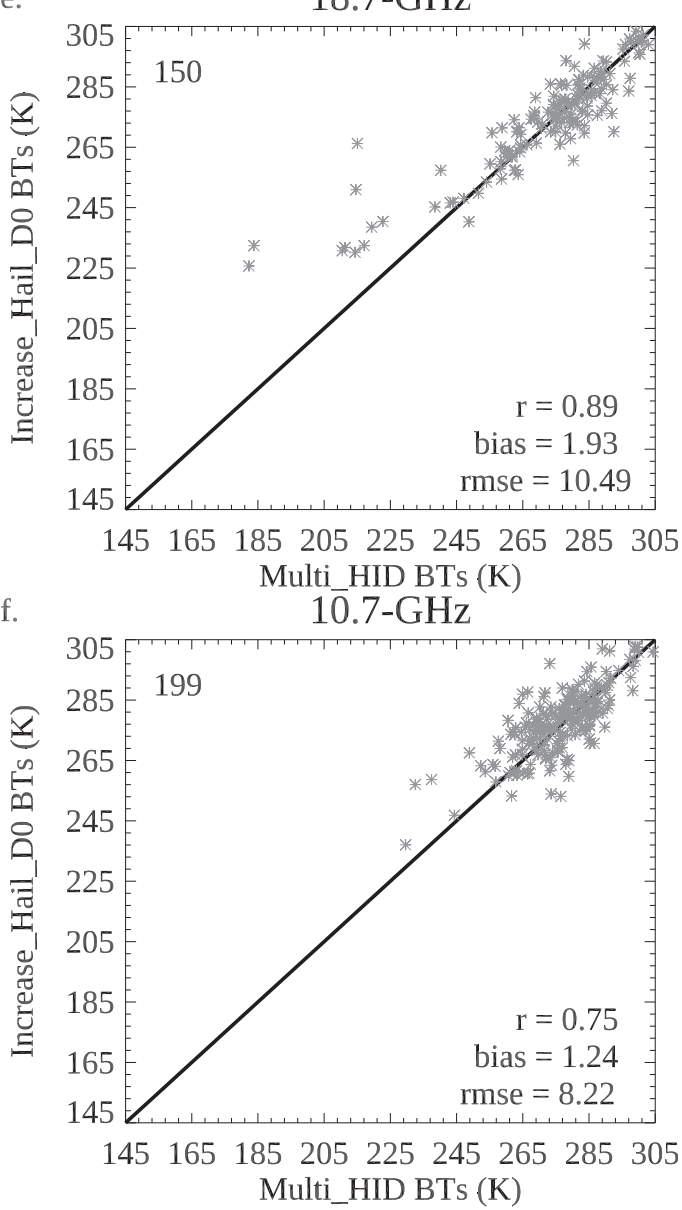

FIG. 12. As in Fig. 11, but showing simulated BTs from Increase_Hail_D0 $\left(D_{o}=18.0 \mathrm{~mm}\right.$ for hail $)$ as a function of simulated BTs from Multi_HID $\left(D_{o}=6.0 \mathrm{~mm}\right.$ for hail). Only pixels that sample hail are included here.

(American Meteorological Society 2019), so it is a minimum hailstone size. The size of $20 \mathrm{~cm}$ is near the record hailstone size observed at the ground (National Weather Association 2010; Allen et al. 2017). Within the area of each $10-\mathrm{GHz}$ GMI pixel, the radar grid points at each level were searched for a beta function value of hail greater than zero. If such a grid point was identified, the maximum $Z_{h}$ within the GMI $10-\mathrm{GHz}$ area was identified. This $Z_{h}$ was used to calculate the number concentration of $0.5-$ or $20-\mathrm{cm}$ hail, and this 
TABLE 5. The minimum observed BT and minimum BT (K) from simulations that contained only $20-$ or $0.5-\mathrm{cm}$ diameter hailstones for various GMI frequencies.

\begin{tabular}{crrr}
\hline \hline Frequency $(\mathrm{GHz})$ & GMI & $20 \mathrm{~cm}$ & $0.5 \mathrm{~cm}$ \\
\hline 183 & 85.9 & 263.9 & 106.2 \\
166 & 82.5 & 277.1 & 100.5 \\
89 & 61.3 & 260.4 & 46.1 \\
36 & 87.0 & 231.4 & 22.1 \\
18 & 154.4 & 237.8 & 33.3 \\
10 & 231.8 & 230.1 & 109.9 \\
\hline
\end{tabular}

number concentration was specified at every radar grid point at that vertical level across the $10-\mathrm{GHz}$ GMI pixel in an attempt to minimize the effects of nonuniform beamfilling. This imposed uniform beamfilling is not realistic and should bias our results toward low BT values, but allows a more direct comparison of the response at different frequencies, independent of their differences in footprint size.

Table 5 shows the minimum BTs from these two simulations as well as the corresponding minimum observed GMI BTs. The minimum BTs produced by the $20-\mathrm{cm}$ simulation are relatively high compared to the minimum observed BTs and those observed over other hailstorms (e.g., Marra et al. 2017) at all frequencies except $10 \mathrm{GHz}$. At $10 \mathrm{GHz}$, the simulated minimum BT of $\sim 230 \mathrm{~K}$ for the 20 - $\mathrm{cm}$ hailstones is similar to the observed value of $231.8 \mathrm{~K}$. In contrast, using $0.5-\mathrm{cm}$ hailstones results in minimum BTs that are much lower than the corresponding $20-\mathrm{cm}$ hailstone BTs at all frequencies and lower than observed at all frequencies $\leq 89 \mathrm{GHz}$. In particular, assuming that $0.5-\mathrm{cm}$ hail is distributed evenly across the satellite pixel results in simulated values as low as 46.1, 22.1, 33.3, and $109.9 \mathrm{~K}$ at $89,36,18$, and $10 \mathrm{GHz}$, respectively. While we have seen $89-\mathrm{GHz}$ BTs this low measured by satellites before, these simulated BTs are far below any we have seen measured at 36-, 18-, or $10-\mathrm{GHz}$ frequencies (cf. Cecil and Chronis 2018). At 183 and $166 \mathrm{GHz}$, the minimum observed BTs are 85.9 and $85.2 \mathrm{~K}$, respectively, about $20 \mathrm{~K}$ colder than the minimum values from the $0.5-\mathrm{cm}$ hail simulation. Results from the Increase_CI_D0 simulation discussed above suggest that the two highest frequencies are most sensitive to cloud ice. Thus, to achieve extremely low BTs at 166 and/or $183 \mathrm{GHz}$ may require the presence of smaller ice crystals in addition to a high concentration of smaller hail or graupel.

Given that the same $Z_{h}$ values were used to calculate the hail concentration for both the $0.5-$ and $20-\mathrm{cm}$ simulations, the maximum number concentration for the smaller hail is 10 orders of magnitude greater than the corresponding maximum for the $20-\mathrm{cm}$ simulation (on the order of $10^{2} \mathrm{~m}^{-3}$ versus $10^{-8} \mathrm{~m}^{-3}$ ). Thus, these results suggest that given the same $Z_{h}$, a greater number of small hailstones can generate a much stronger scattering signature at all frequencies than a few large hailstones. That is not to say a relationship between particle size or concentration and BT is monotonic. Another simulation assuming 4-cm diameter hail (not shown) gave very similar minimum BTs as the $20-\mathrm{cm}$ simulation at 183,166 , and $10 \mathrm{GHz}$. The $89-\mathrm{GHz}$ minimum BT was $8 \mathrm{~K}$ lower and the 36- and $18-\mathrm{GHz}$ BT were $12 \mathrm{~K}$ higher for the $4-\mathrm{cm}$ simulation than for the $20-\mathrm{cm}$ simulation. For all frequencies, the $4-\mathrm{cm}$ simulation gave BTs much closer to those from the $20-\mathrm{cm}$ simulation than from the $0.5-\mathrm{cm}$ simulation.

\section{Reducing differences between simulated and observed BTs}

Emission and scattering from hydrometeors depend on many factors that interact in complicated ways. In addition, several sources of uncertainty (e.g., horizontally inhomogeneous atmosphere, unknown form of PSDs, unknown particle density or variations in density across domain, complex particle shapes, etc.) ensure that simulated BTs will never perfectly match observed values. Nevertheless, several additional simulations were conducted based on the results described above to try to bring the simulated BTs closer to those observed. Specifically, the decrease in simulated scattering that occurs with an increase in $D_{o}$ and vice versa was used in an attempt to reduce the scattering in the anvil region and increase scattering in the hail hole observed at the two highest frequencies in the control simulation (Fig. 2). For the Adjust_Ice_D0 simulation, $D_{o}$ of cloud ice was increased to $0.4 \mathrm{~mm}$, while $D_{o}$ for hail and graupel was decreased relative to Multi_HID (Table 3).

The resulting BTs from Adjust_Ice_D0 are plotted for various frequencies in Figs. 13-15. The corresponding Multi_HID and GMI BTs are reproduced from Figs. 2-4 for convenience. At 183 and $166 \mathrm{GHz}$ (Fig. 13), the scattering is generally reduced in the eastern anvil region for Adjust_Ice_D0 relative to Multi_HID in better agreement with GMI BTs. Nevertheless, an area of relatively low BTs still exists centered near $33.1^{\circ} \mathrm{N}$, $97.7^{\circ} \mathrm{W}$ for simulated BTs that is not present in the observed BTs. BTs in the hail hole are reduced for the Adjust_Ice_D0 simulation with magnitudes similar to those observed at $166 \mathrm{GHz}$ but too cold at $183 \mathrm{GHz}$. Around the hail hole over the southern convective cell, Adjust_Ice_D0 BTs are still too low relative to GMI BTs, and scattering over the core of the other two convective cells is still too strong. In addition, the area of cooler BTs in the north-central part of the domain 
a. Adjust_Ice_D0 183.3 $\pm 7-\mathrm{GHz}(52.4 \mathrm{~K})$

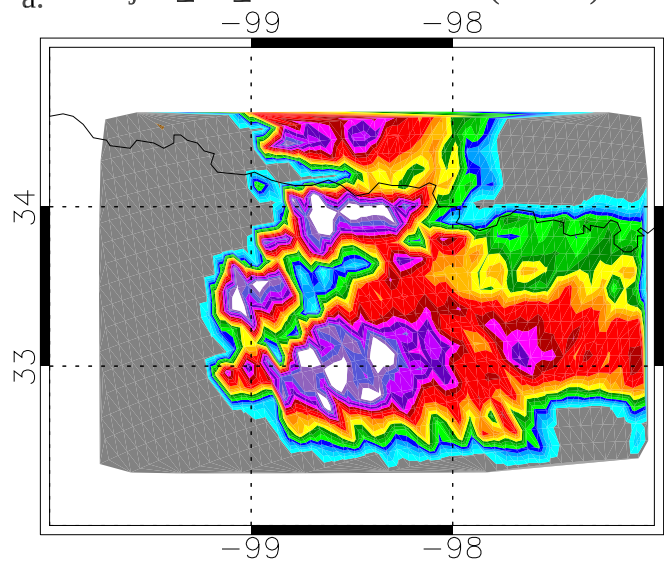

b. Multi_HID $183.3 \pm 7-\mathrm{GHz}(50.4 \mathrm{~K})$
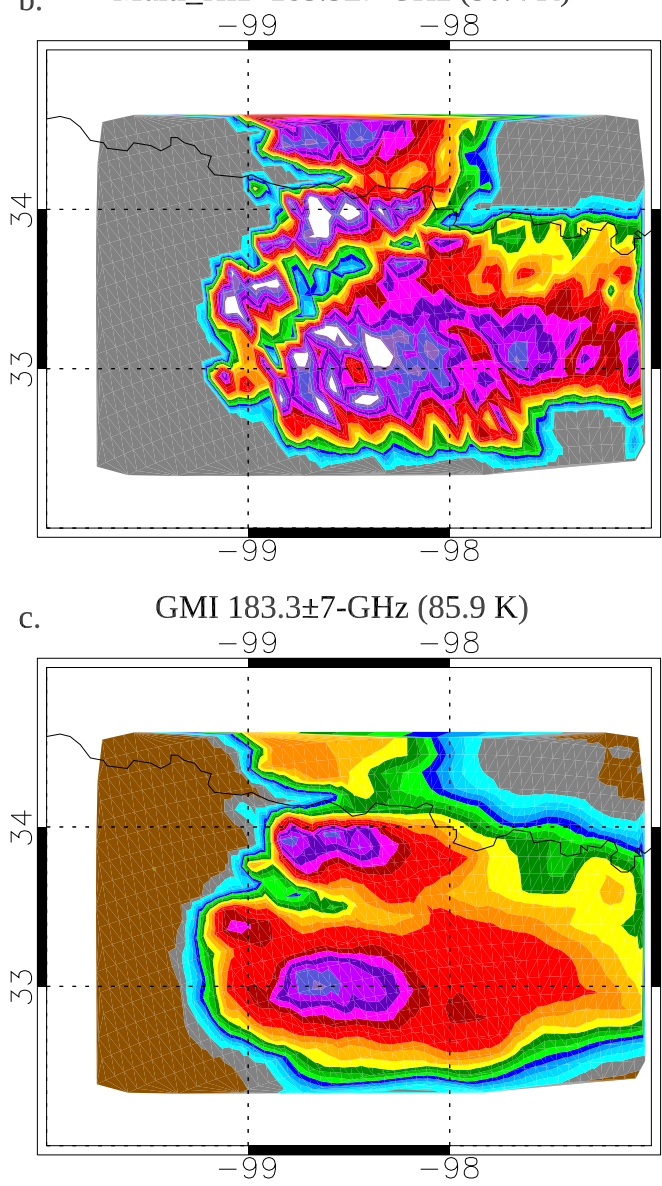

d. Adjust_Ice_D0 166.0-GHz (54.0 K)

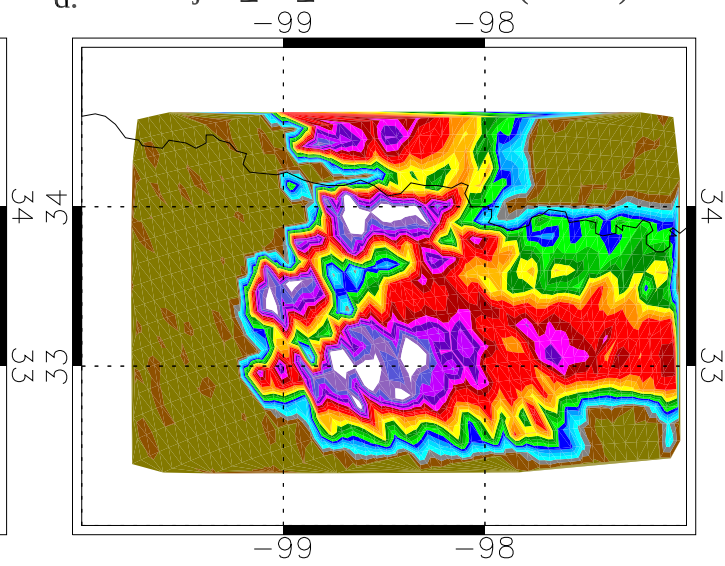

e. Multi_HID $166.0-\mathrm{GHz}(54.9 \mathrm{~K})$

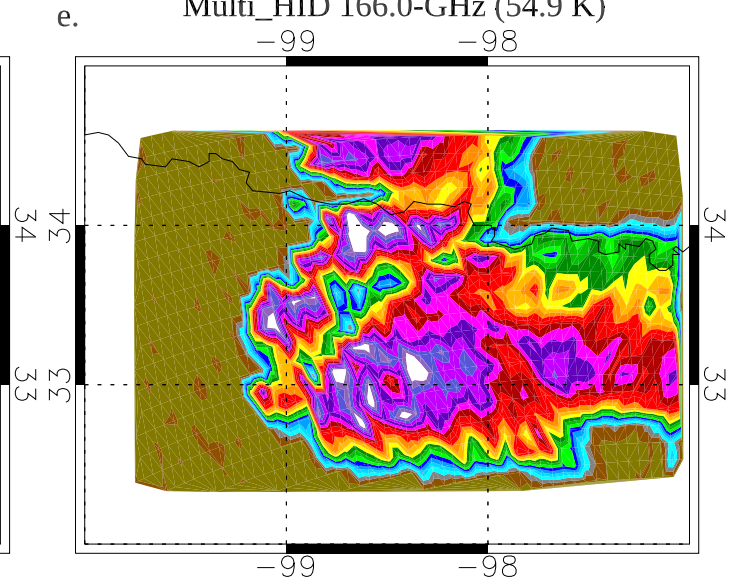

f.
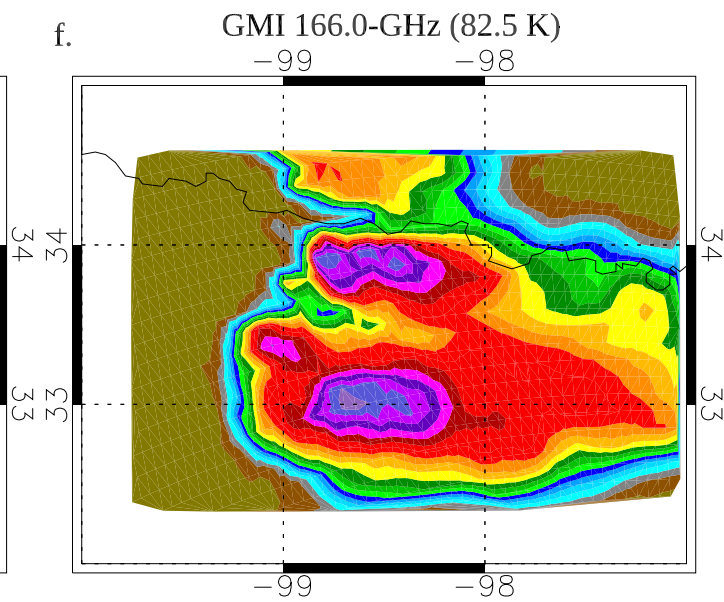

Brightness Temperature (K)

$\begin{array}{lllllllllllllllllllll}80 & 90 & 100 & 110 & 120 & 130 & 140 & 150 & 160 & 170 & 180 & 190 & 200 & 210 & 220 & 230 & 240 & 250 & 260 & 270 & 280\end{array}$

FIG. 13. The $183-\mathrm{GHz}$ (a) simulated BTs where $D_{o}$ of cloud ice is increased while $D_{o}$ of large ice species (lowdensity graupel, high-density graupel, and hail) is decreased relative to Multi_HID (Table 3; Adjust_Ice_D0), (b) simulated BTs from the control simulation (Multi_HID), and (c) observed BTs. (d)-(f) As in (a)-(c), but for $166 \mathrm{GHz}$. The minimum BT in each panel is given in the title of each panel. 
a. Adjust_Ice_D0 89.0-GHz (70.9 K)

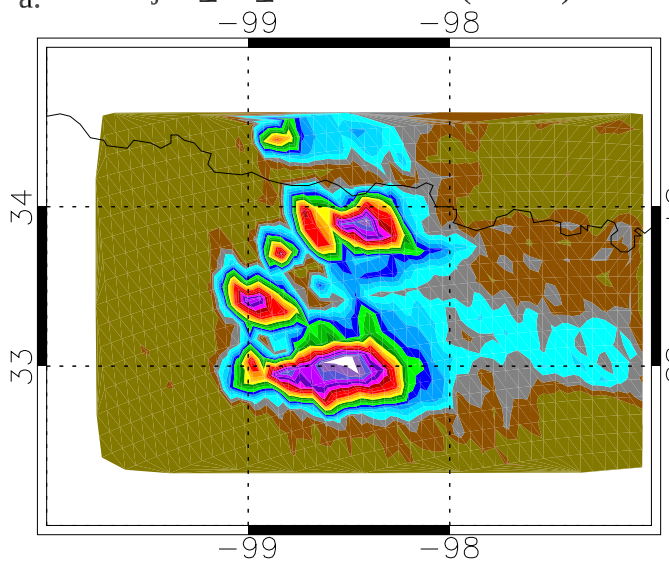

b. Multi_HID 89.0-GHz (93.0 K)
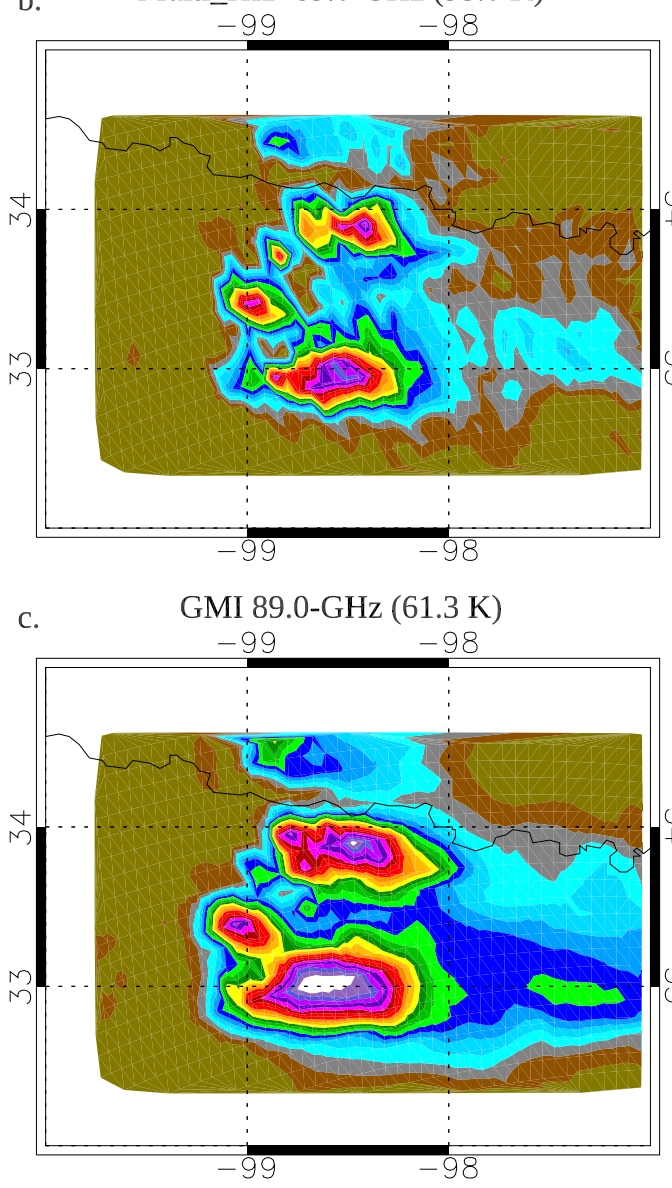

d. Adjust_Ice_D0 36.5-GHz (117.8 K)

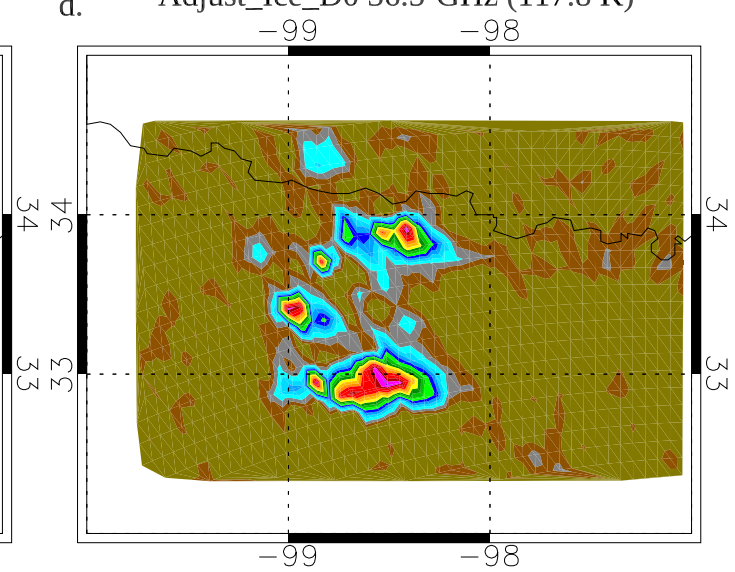

e. Multi_HID 36.5-GHz (126.1 K)

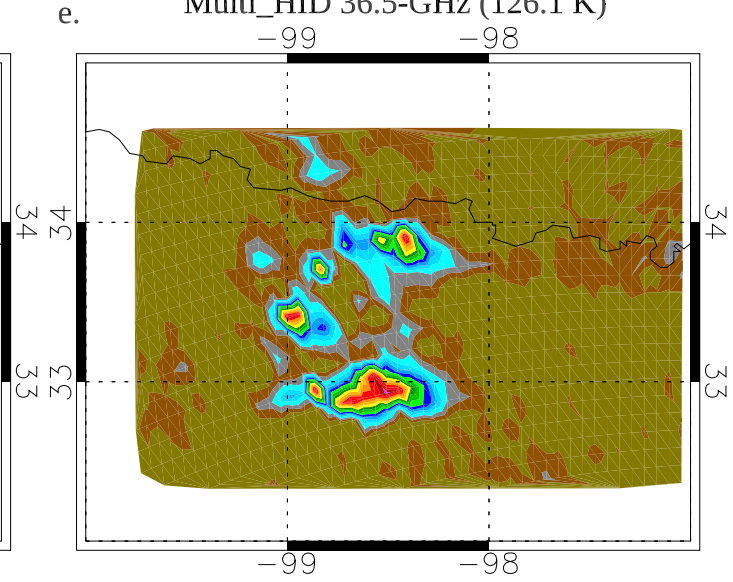

f. f. GMI 36.5-GHz (87.0 K)

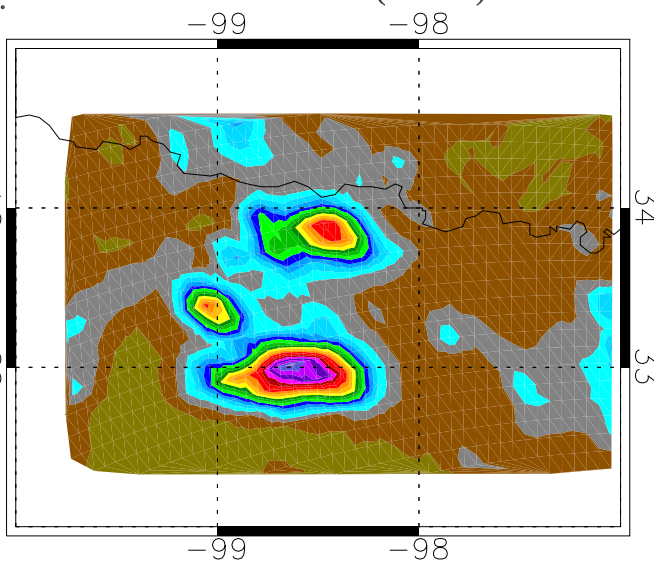

Brightness Temperature (K)

$\begin{array}{lllllllllllllllllllll}80 & 90 & 100 & 110 & 120 & 130 & 140 & 150 & 160 & 170 & 180 & 190 & 200 & 210 & 220 & 230 & 240 & 250 & 260 & 270 & 280\end{array}$

FIG. 14. As in Fig. 13, but for 89 and $36 \mathrm{GHz}$. 
a. Adjust_Ice_D0 18.7-GHz (173.5 K)

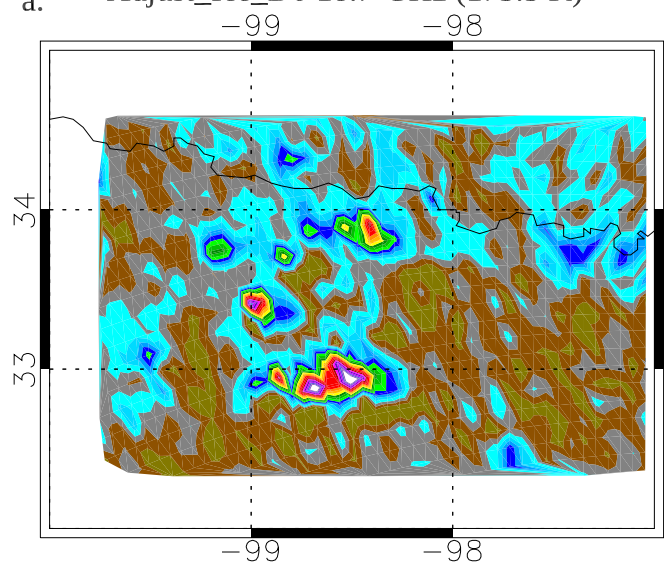

b. Multi_HID 18.7-GHz (182.3 K)
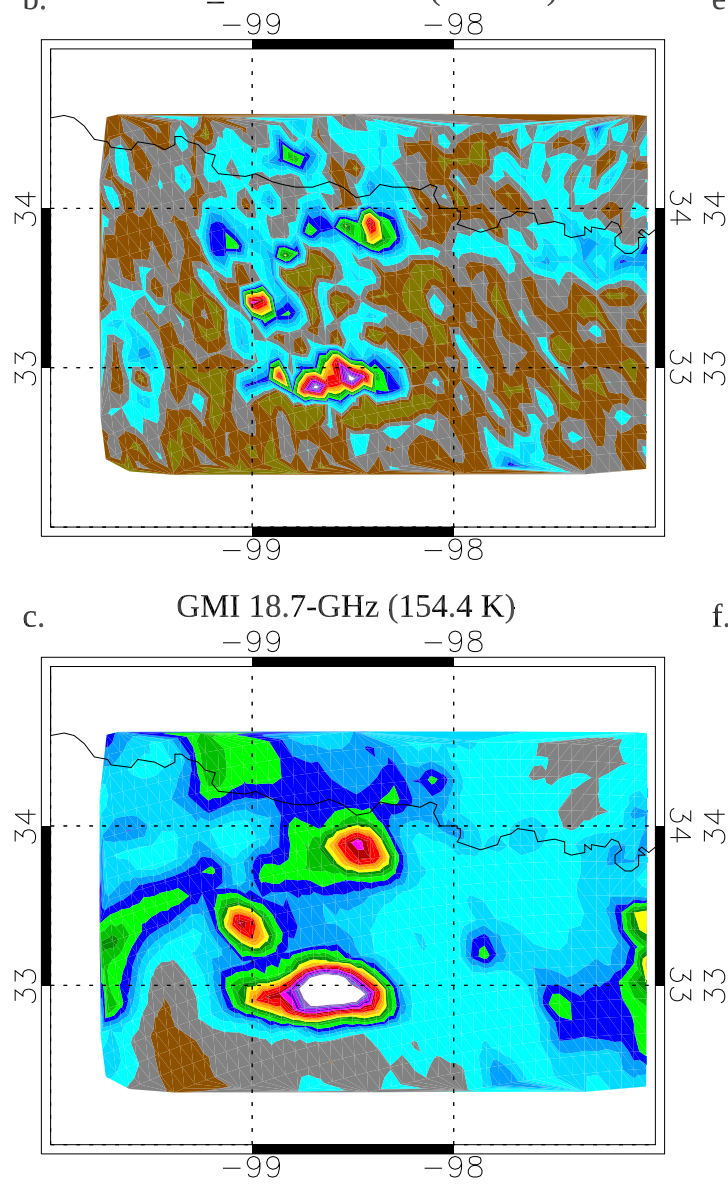

d. Adjust_Ice_D0 10.7-GHz (227.3 K)

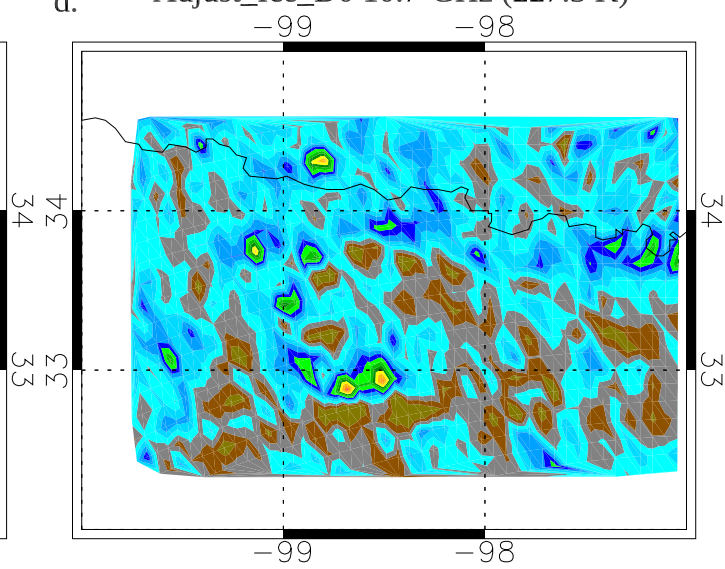

e. Multi_HID 10.7-GHz (229.6 K)

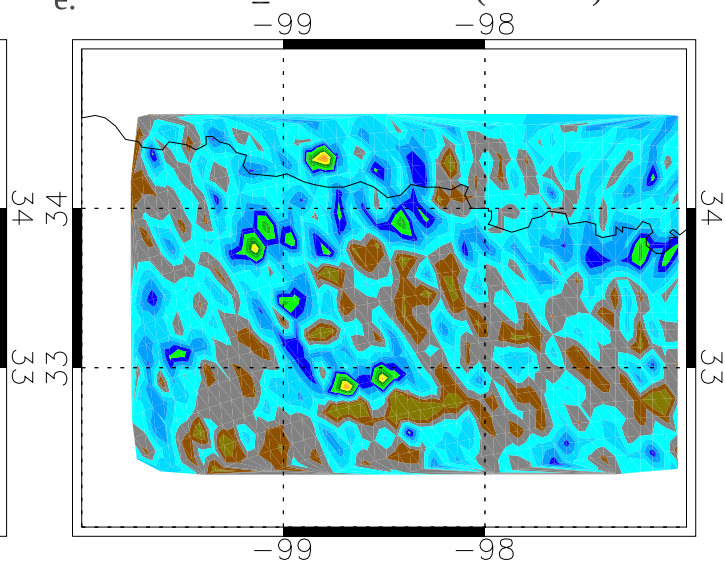

f.
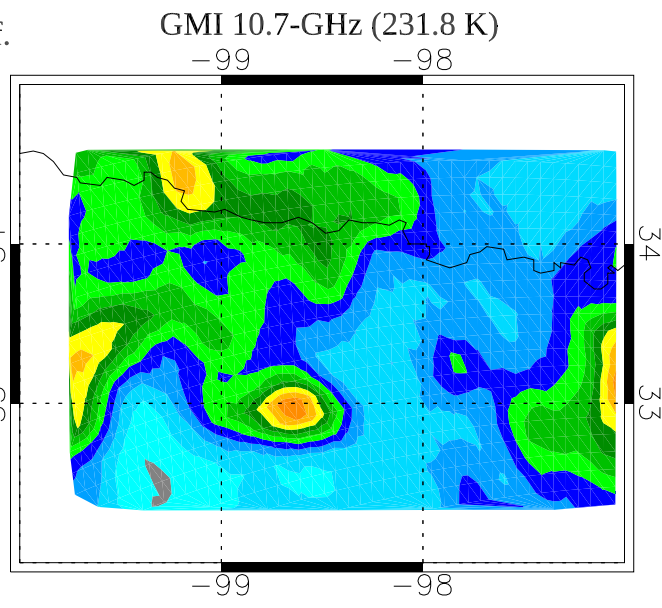

Brightness Temperature (K)

$190195200205210215220 \quad 225230235240245250255260 \quad 265270275280285290$ FIG. 15. As in Fig. 13, but for 18 and $10 \mathrm{GHz}$. 
dips down to around $160 \mathrm{~K}$ in observations, but simulated BTs for both simulations extend down to $90 \mathrm{~K}$ at the two highest frequencies. However, the area of this excessive scattering is slightly reduced for Adjust_Ice_D0 relative to Multi_HID.

At $36 \mathrm{GHz}$ (Fig. 14), simulated BTs for Adjust_Ice_D0 show little change relative to Multi_HID BTs. Larger differences are observed at $89 \mathrm{GHz}$. In particular, Adjust_Ice_D0 exhibits stronger scattering in the convective cores relative to Multi_HID in better agreement with observed BTs. The area with the lowest BTs also appears to better match that of observed BTs. However, the BTs in the anvil region to the east for Adjust_Ice_D0 at $89 \mathrm{GHz}$ are still too warm relative to GMI values. At 18 and $10 \mathrm{GHz}$, changing PSD parameters for various hydrometeor species individually resulted in virtually no change in simulated BTs. Hence, it is not surprising that the maps of BTs at these frequencies for Adjust_Ice_D0 look nearly identical to those of Multi_HID (Fig. 15) and do not show any better agreement with observed BTs.

Table 6 shows statistics for the comparison between observed BTs and simulated BTs from Adjust_Ice_D0 that can be compared with the corresponding statistics for Multi_HID shown in Table 4. The bias and RMSE for Adjust_Ice_D0 are improved relative to the control simulation for the three highest frequencies with the magnitude of the improvement generally increasing with frequency. Consistent with what is observed from the BT maps, adjusting $D_{o}$ for multiple frozen hydrometeor species has little impact on the three lowest frequencies, with the correlation coefficient, bias, and RMSE of Adjust_Ice_D0 very similar to those of Multi_HID.

In summary, changing $D_{o}$ of various frozen hydrometeor species results in better agreement with observed BTs in some respects but little change in other areas. In agreement with simulations where PSD parameters were changed for each hydrometeor type individually, increasing $D_{o}$ of cloud ice results in less scattering at the two highest frequencies in areas where that hydrometeor type would be expected to be dominant (i.e., eastern anvil region). Decreasing $D_{o}$ of hail and graupel results in stronger scattering at frequencies $\geq 89 \mathrm{GHz}$ where these hydrometeor types are present in the core of the southern convective cell. Stronger scattering is also observed where hail and graupel are present in the cores of the other two cells for $89 \mathrm{GHz}$, but little change is observed in these areas for the two highest frequencies. Perhaps the impact of ice crystals above the hail/graupel is dominating any influence of changing the PSD of the larger ice species for the two highest frequencies.

\section{Summary and conclusions}

In this study, ARTS radiative transfer simulations of a hailstorm at 2225 UTC 26 May 2015 near Dallas, Texas, are used to better understand how simulated GMI BTs respond to changing PSD parameters of various hydrometeor types for a given $Z_{h}$ and to better understand what characteristic (e.g., size, number concentration) of hydrometeor PSDs is most important for generating extremely low observed BTs (e.g., Marra et al. 2017). Results suggest that increasing $D_{o}$ of a normalized gamma distribution of ice crystals, low-density graupel, high-density graupel, or hail leads to warmer BTs (i.e., a weaker scattering signature) at various frequencies depending on hydrometeor type. The 166- and $183-\mathrm{GHz}$ frequencies are most sensitive to changing the PSD of small ice crystals, which is generally consistent with previous work (e.g., Bennartz and Bauer 2003; Hong et al. 2005). Frequencies $\geq 36 \mathrm{GHz}$ respond to changing graupel PSDs, although at $36 \mathrm{GHz}$, this sensitivity is limited to changing the high-density graupel distribution (with less sensitivity to low-density graupel). Changing the hail distribution seems to only cause a response at 18 and $36 \mathrm{GHz}$ generally consistent with numerous hail detection studies (e.g., Cecil 2009; Leppert and Cecil 2015; Mroz et al. 2017).

Previous work (e.g., Galligani et al. 2013) has shown that scattering tends to increase with increasing particle size. However, when holding $Z_{h}$ (or hydrometeor mass) constant, Bennartz and Petty (2001) showed a reduction in scattering as size is increased. Increasing $D_{o}$ results in smaller number concentrations under the fixed $Z_{h}$ or fixed mass constraint. Specifically, fewer but larger frozen hydrometeors present a smaller surface area for scattering, resulting in warmer BTs (Hartmann 1994). Therefore, given the same $Z_{h}$ or mass, a larger number of smaller particles may generate a stronger scattering signature than a small number of larger hydrometeors. In other words, number concentration appears to be more important than size for scattering when $Z_{h}$ or mass is held constant.

Results not shown here suggest that simulated GMI BTs for this severe thunderstorm case are not sensitive to changes in $\mu$ of the PSD of any hydrometeor type. In addition, output of simulations where all hydrometeor types were included suggest little sensitivity of BTs at any frequency to changing any PSD parameter of rain, big drops, or snow. The emission signal of liquid hydrometeor types can be important over ocean (radiometrically cold background) at lower frequencies (e.g., Wilheit et al. 1991). However, the radiometrically warm background of land (used here) provides relatively little distinction from the emission from liquid in a cloud. 
TABLE 6. The correlation coefficient $r$, bias (K), and RMSE (K) for the comparison of Adjust_Ice_D0 simulated BTs to the corresponding observed BTs as a function of frequency.

\begin{tabular}{ccrc}
\hline \hline Frequency $(\mathrm{GHz})$ & $r$ & \multicolumn{1}{c}{ Bias } & RMSE \\
\hline 183 & 0.86 & -1.42 & 31.79 \\
166 & 0.88 & 1.24 & 32.29 \\
89 & 0.87 & 17.36 & 28.78 \\
36 & 0.79 & 13.27 & 21.51 \\
18 & 0.59 & 12.17 & 17.68 \\
10 & 0.23 & 14.85 & 18.74 \\
\hline
\end{tabular}

In addition, ice above liquid has a tendency to obscure the signal from the liquid layer below (Hong et al. 2005), particularly in the type of case used here. The lack of a response to changing the snow PSD, especially at higher frequencies, is likely due to the masking influence of cloud ice. Simulations conducted with only snow do show a relatively weaker scattering signature at 166 and $183 \mathrm{GHz}$, compared to simulations with only cloud ice.

The 18- and 36-GHz channels showed some sensitivity to changing the PSD (i.e., $D_{o}$ ) for hail. However, the signal was not as clear and strong as may be expected. Further analysis suggested that nonuniform beamfilling by hail may explain this relatively weak signal. Simulations that utilized only a single size bin for hail ( 0.5 or $20 \mathrm{~cm}$ ) where hail number concentrations were spread evenly across the area of a $10-\mathrm{GHz}$ GMI pixel indicated a much stronger scattering signature at all frequencies for the $0.5-\mathrm{cm}$ hail compared to the $20-\mathrm{cm}$ hail. Both simulations used the same $Z_{h}$ causing the concentration of $0.5-\mathrm{cm}$ hail to be much higher than that of the $20-\mathrm{cm}$ hail. Thus, the results of these simulations with a single size of hail suggest that given some $Z_{h}$ from hail, lower BTs will result if the hail is distributed among a large number of smaller hailstones compared to few large ones. Given these extreme (but realistic) sizes of hail and associated concentrations, simulated BTs were as low as $106.2,100.5,46.1,22.1,33.3$, and $109.9 \mathrm{~K}$ at 183 , $166,89,36,18$, and $10 \mathrm{GHz}$, respectively, using the $0.5-\mathrm{cm}$ hailstones. At frequencies $\leq 89 \mathrm{GHz}$, these simulated BTs are much lower than in observations, but the simulated BTs at the two highest frequencies are $\sim 20 \mathrm{~K}$ warmer than the minimum observed here. Thus, at the two highest frequencies, the presence of additional hydrometeor types (i.e., small ice crystals) in addition to a large concentration of hail may be required to achieve extremely low BTs.

The simulation representing giant hailstones produced such high BTs at frequencies $\geq 89 \mathrm{GHz}$ that those BT values would not even stand out as noteworthy deep convection if encountered in observations. The simulated 36-GHz BT is near thresholds used by Mroz et al. (2017) and Ni et al. (2017) for hail detection, but well above the threshold used by Cecil and Blankenship (2012). The simulated 18- and $10-\mathrm{GHz}$ BTs are consistent with those observed above intense thunderstorms. The simulated $10-36-\mathrm{GHz}$ BTs from the small hail test, on the other hand, are so low they would likely be interpreted as some sort of corrupted data if observed by a satellite. The general result that an extremely low BT is more indicative of a large number of hailstones than a large size of hailstones seems to run counter to empirical relationships between BT and hailstone size. A key distinction is that lower BT together with increased hailstone size can be accompanied by increased $Z_{h}$, whereas our simulations made comparisons where $Z_{h}$ was held constant. The conclusion that an extremely low BT can also result from a large number of small hailstones brings to mind cases with deep hail accumulations on the ground, also known as "plowable hail" (Kalina et al. 2016).

The normalized gamma distribution was used here because that is the distribution used for the combined DPR-GMI 2BCMB product. However, future work may test the sensitivity to different forms of the PSD for different hydrometeor types (e.g., exponential). In addition, tests could be run for different particle shapes, densities, aspect ratios, orientations, etc. This study was generally limited to changing the parameters of one particular form of the PSD given the large computational expense of single-scattering calculations. Using various particle shapes, densities, etc., would require numerous iterations of single-scattering calculations.

Acknowledgments. Funding for this research was graciously provided through the NASA Precipitation Measurement Missions Science Team (Grant NNX16AH29G). The authors wish to thank Dr. Brenda Dolan for providing the code used for the HID, Dr. S. Joseph Munchak for providing the PyARTS code and scripts that were instrumental in helping to run PyARTS as well as ARTS, and three anonymous reviewers who helped to improve the manuscript. The authors also wish to gratefully acknowledge the NOAA National Centers for Environmental Information for providing the WSR-88D data, the University of Wyoming for providing the sounding data (available from http://weather.uwyo.edu/upperair/sounding.html), the National Centers for Atmospheric Research for providing the Radx code, the University of Hamburg for providing the ARTS code, and NASA's Precipitation Processing System for providing the GMI data.

\section{REFERENCES}

Adler, R. F., H.-Y. M. Yeh, N. Prasad, W.-K. Tao, and J. Simpson, 1991: Microwave simulations of a tropical rainfall system 
with a three-dimensional cloud model. J. Appl. Meteor., 30, 924-953, https://doi.org/10.1175/1520-0450-30.7.924.

Allen, J. T., M. K. Tippett, Y. Kaheil, A. H. Sobel, C. Lepore, S. Nong, and A. Muehlbauer, 2017: An extreme value model for U.S. hail size. Mon. Wea. Rev., 145, 4501-4519, https:// doi.org/10.1175/MWR-D-17-0119.1.

American Meteorological Society, 2019: Graupel. Glossary of Meteorology, http://glossary.ametsoc.org/wiki/Graupel.

Bennartz, R., and G. W. Petty, 2001: The sensitivity of microwave remote sensing observations of precipitation to ice particle size distributions. J. Appl. Meteor., 40, 345-364, https://doi.org/ 10.1175/1520-0450(2001)040<0345:TSOMRS > 2.0.CO;2.

- and P. Bauer, 2003: Sensitivity of microwave radiances at 85 $183 \mathrm{GHz}$ to precipitating ice particles. Radio Sci., 38, 8075, https://doi.org/10.1029/2002RS002626.

Buehler, S. A., J. Mendrok, P. Eriksson, A. Perrin, R. Larson, and O. Lemke, 2018: ARTS, the Atmospheric Radiative Transfer Simulator-version 2.2, the planetary toolbox edition. Geosci. Model Dev., 11, 1537-1556, https://doi.org/10.5194/ gmd-11-1537-2018.

Casella, D., A. Mugnai, P. Sano, and M. Formenton, 2008: Microwave single-scattering properties of randomly oriented soft-ice hydrometeors. Adv. Geosci., 17, 79-85, https://doi.org/ 10.5194/adgeo-17-79-2008.

Cecil, D. J., 2009: Passive microwave brightness temperatures as proxies for hailstorms. J. Appl. Meteor. Climatol., 48, 12811286, https://doi.org/10.1175/2009JAMC2125.1.

_ , and C. B. Blankenship, 2012: Toward a global climatology of severe hailstorms as estimated by satellite passive microwave imagers. J. Climate, 25, 687-703, https://doi.org/10.1175/ JCLI-D-11-00130.1.

_ , and T. Chronis, 2018: Polarization-corrected temperatures for 10-, 19-, 37-, and 89-GHz passive microwave frequencies. J. Appl. Meteor. Climatol., 57, 2249-2265, https://doi.org/ 10.1175/JAMC-D-18-0022.1.

Dolan, B., and S. A. Rutledge, 2009: A theory-based hydrometeor identification algorithm for X-band polarimetric radars. J. Atmos. Oceanic Technol., 26, 2071-2088, https://doi.org/10.1175/ 2009JTECHA1208.1.

, S. Lim, V. Chandrasekar, and M. Thurai, 2013: A robust $\mathrm{C}$-band hydrometeor identification algorithm and application to a long-term polarimetric radar dataset. J. Appl. Meteor. Climatol., 52, 2162-2186, https://doi.org/10.1175/ JAMC-D-12-0275.1.

Eriksson, P., S. A. Buehler, C. P. Davis, C. Emde, and O. Lemke, 2011: ARTS, the Atmospheric Radiative Transfer Simulator, version 2. J. Quant. Spectrosc. Radiat. Transfer, 112, 15511558, https://doi.org/10.1016/j.jqsrt.2011.03.001.

Ferraro, R. R., and G. F. Marks, 1995: The development of SSM/I rainrate retrieval algorithms using ground-based radar measurements. J. Atmos. Oceanic Technol., 12, 755-770, https://doi.org/ 10.1175/1520-0426(1995)012<0755:TDOSRR > 2.0.CO;2.

_- J. Beauchamp, D. J. Cecil, and G. M. Heymsfield, 2015: A prototype hail detection algorithm and hail climatology developed with the Advanced Microwave Sounding Unit (AMSU). Atmos. Res., 163, 24-35, https://doi.org/10.1016/ j.atmosres.2014.08.010.

Galligani, V. S., C. Prigent, E. Defer, C. Jimenez, and P. Eriksson, 2013: The impact of the melting layer on the passive microwave cloud scattering signal observed from satellites: A study using TRMM microwave passive and active measurements. J. Geophys. Res. Atmos., 118, 5667-5678, https://doi.org/ 10.1002/JGRD.50431.
Grecu, M., W. S. Olson, S. J. Munchak, S. Ringerud, L. Liao, Z. Haddad, B. L. Kelley, and S. F. McLaughlin, 2016: The GPM combined algorithm. J. Atmos. Oceanic Technol., 33, 2225-2245, https://doi.org/10.1175/JTECH-D-16-0019.1.

Hartmann, D. L., 1994: Global Physical Climatology. Academic Press, $411 \mathrm{pp}$

Hogan, R. J., R. Honeyager, J. Tyynela, and S. Kneifel, 2017: Calculating the millimetre-wave scattering phase function of snowflakes using the self-similar Rayleigh-Gans approximation. Quart. J. Roy. Meteor. Soc., 143, 834-844, https://doi.org/ 10.1002/qj.2968.

Hong, G., G. Heygster, J. Miao, and K. Kunzi, 2005: Sensitivity of microwave brightness temperatures to hydrometeors in a tropical deep convective cloud system at 89-190 GHz. Radio Sci., 40, RS4003, https://doi.org/10.1029/2004RS003129.

Hou, A. Y., and Coauthors, 2014: The Global Precipitation Measurement (GPM) mission. Bull. Amer. Meteor. Soc., 95, 701722, https://doi.org/10.1175/BAMS-D-13-00164.1.

Kalina, E. A., K. Friedrich, B. C. Motta, W. Deierling, G. T. Stano, and N. N. Rydell, 2016: Colorado plowable hailstorms: Synoptic weather, radar, and lightning characteristics. Wea. Forecasting, 31, 663-693, https://doi.org/10.1175/WAF-D-15-0037.1.

Kim, M.-J., M. S. Kulie, C. O'Dell, and R. Bennartz, 2007: Scattering of ice particles at microwave frequencies: A physically based parameterization. J. Appl. Meteor. Climatol., 46, 615633, https://doi.org/10.1175/JAM2483.1.

Kulie, M. S., R. Bennartz, T. J. Greenwald, Y. Chen, and F. Weng, 2010: Uncertainties in microwave properties of frozen precipitation: Implications for remote sensing and data assimilation. J. Atmos. Sci., 67, 3471-3487, https://doi.org/10.1175/ 2010JAS3520.1.

Leppert, K. D., II, and D. J. Cecil, 2015: Signatures of hydrometeor species from airborne passive microwave data for frequencies 10-183 GHz. J. Appl. Meteor. Climatol., 54, 1313-1334, https:// doi.org/10.1175/JAMC-D-14-0145.1.

Marra, A. C., and Coauthors, 2017: Observational analysis of an exceptionally intense hailstorm over the Mediterranean area: Role of the GPM Core Observatory. Atmos. Res., 192, 72-90, https://doi.org/10.1016/j.atmosres.2017.03.019.

McGaughey, G., E. J. Zipser, R. W. Spencer, and R. E. Hood, 1996: High-resolution passive microwave observations of convective systems over the tropical Pacific Ocean. J. Appl. Meteor., 35, 1921-1947, https://doi.org/10.1175/1520-0450(1996)035<1921: HRPMOO $>2.0 . \mathrm{CO} ; 2$.

Meirold-Mautner, I., C. Prigent, E. Defer, J. R. Pardo, J.-P. Chaboureau, J.-P. Pinty, M. Mech, and S. Crewell, 2007: Radiative transfer simulations using mesoscale cloud model outputs: Comparisons with passive microwave and infrared satellite observations for midlatitudes. J. Atmos. Sci., 64, 15501568, https://doi.org/10.1175/JAS3896.1.

Mishchenko, M. I., 1991: Extinction and polarization of transmitted light by partially aligned nonspherical grains. Astrophys. J., 367, 561-574, https://doi.org/10.1086/169652.

- 2000: Calculation of the amplitude matrix for a nonspherical particle in a fixed orientation. Appl. Opt., 39, 1026-1031, https://doi.org/10.1364/AO.39.001026.

— , and L. D. Travis, 1998: Capabilities and limitations of a current FORTRAN implementation of the T-matrix method for randomly oriented, rotationally symmetric scatterers. J. Quant. Spectrosc. Radiat. Transfer, 60, 309-324, https:// doi.org/10.1016/S0022-4073(98)00008-9.

Mroz, K., A. Battaglia, T. J. Lang, D. J. Cecil, S. Tanelli, and F. Tridon, 2017: Hail-detection algorithm for the GPM Core 
Observatory satellite sensors. J. Appl. Meteor. Climatol., 56, 1939-1957, https://doi.org/10.1175/JAMC-D-16-0368.1.

NASA, 2016: Precipitation Processing System TRMM/GPM Public Data Archive, version 5. NASA Goddard Space Flight Center, accessed 5 August 2019, https://pps.gsfc.nasa.gov/ ppsindex.html.

National Centers for Environmental Information, 2015: NEXRAD Data Archive, Inventory and Access, version 6. NOAA, accessed 5 August 2019, https://www.ncdc.noaa.gov/nexradinv/.

National Weather Association, 2010: New national hail records set in South Dakota. National Weather Association Newsletter, No. 10-7, National Weather Association, Raleigh, NC, 1, http://nwafiles.nwas.org/newsletters/pdf/news_jul2010.pdf.

Ni, X., C. Liu, D. J. Cecil, and Q. Zhang, 2017: On the detection of hail using satellite passive microwave radiometers and precipitation radar. J. Appl. Meteor. Climatol., 56, 2693-2709, https://doi.org/10.1175/JAMC-D-17-0065.1.

Olson, W. S., and H. Masunaga, 2018: GPM combined radarradiometer precipitation Algorithm Theoretical Basis Document (version 2). EORC/JAXA, 58 pp., http://www.eorc.jaxa.jp/ GPM/doc/algorithm/GPM2011CombinedL2ATBD.pdf.

_, and Coauthors, 2016: The microwave radiative properties of falling snow derived from nonspherical ice particle models. Part II: Initial testing using radar, radiometer and in situ observations. J. Appl. Meteor. Climatol., 55, 709-722, https:// doi.org/10.1175/JAMC-D-15-0131.1.

Skofronick-Jackson, G., and Coauthors, 2017: The Global Precipitation Measurement (GPM) mission for science and society. Bull. Amer. Meteor. Soc., 98, 1679-1695, https://doi.org/ 10.1175/BAMS-D-15-00306.1.

Smith, E. A., H. J. Cooper, X. Xiang, A. Mugnai, and G. J. Tripoli, 1992: Foundations for statistical-physical precipitation retrieval from passive microwave satellite measurements. Part I: Brightness temperature properties of a time-dependent cloudradiation model. J. Appl. Meteor., 31, 506-531, https://doi.org/ 10.1175/1520-0450(1992)031<0506:FFSPPR >2.0.CO;2.

Spencer, R. W., and D. A. Santek, 1985: Measuring the global distribution of intense convection over land with passive microwave radiometry. J. Climate Appl. Meteor., 24,
860-864, https://doi.org/10.1175/1520-0450(1985)024<0860: MTGDOI $>2.0 . \mathrm{CO} ; 2$.

_ W. W. Olson, W. Rongzhang, D. W. Martin, J. A. Weinman, and D. A. Santek, 1983: Heavy thunderstorms observed over land by the Nimbus 7 scanning multichannel microwave radiometer. J. Climate Appl. Meteor., 22, 1041-1046, https://doi.org/ 10.1175/1520-0450(1983)022<1041:HTOOLB > 2.0.CO;2.

—, H. M. Goodman, and R. E. Hood, 1989: Precipitation retrieval over land and ocean with the SSM/I: Identification and characteristics of the scattering signal. J. Atmos. Oceanic Technol., 6, 254-273, https://doi.org/10.1175/1520-0426(1989) 006<0254:PROLAO >2.0.CO;2.

Storm Prediction Center, 2015: Storm Reports. NOAA, accessed 5 August 2019, https://www.spc.noaa.gov/climo/reports/ 150526_rpts.html.

Tang, G., P. Yang, P. G. Stegmann, R. L. Panetta, L. Tsang, and B. Johnson, 2017: Effect of particle shape, density, and inhomogeneity on the microwave optical properties of graupel and hailstones. IEEE Trans. Geosci. Remote Sens., 55, 6366-6378, https://doi.org/10.1109/ TGRS.2017.2726994.

Vivekanandan, J., J. Turk, and V. N. Bringi, 1991: Ice water path estimation and characterization using passive microwave radiometry. J. Appl. Meteor., 30, 1407-1421, https://doi.org/ 10.1175/1520-0450(1991)030<1407:IWPEAC>2.0.CO;2.

Wilheit, T. T., A. T. C. Chang, M. S. V. Rao, E. B. Rodgers, and J. S. Theon, 1977: A satellite technique for quantitatively mapping rainfall rates over the oceans. J. Appl. Meteor., 16, 551-560, https://doi.org/10.1175/1520-0450(1977)016<0551: ASTFQM $>2.0 . \mathrm{CO} ; 2$.

$\longrightarrow, \ldots$, and L. S. Chiu, 1991: Retrieval of monthly rainfall indices from microwave radiometric measurements using probability distribution functions. J. Atmos. Oceanic Technol., 8, 118-136, https://doi.org/10.1175/1520-0426(1991)008<0118: ROMRIF $>2.0 . \mathrm{CO} ; 2$.

You, Y., N.-Y. Wang, R. Ferraro, and S. Rudlosky, 2017: Quantifying the snowfall detection performance of the GPM Microwave Imager channels over land. J. Hydrometeor., 18, 729-751, https://doi.org/10.1175/JHM-D-16-0190.1. 